\title{
Self-similar dynamics of two-phase flows injected into a confined porous layer
}

\author{
ZHONG ZHENG ${ }^{1,2,3,4} \dagger$ AND JEROMEA. NEUFELD $\mathbf{D}^{1,2,3}$ \\ ${ }^{1}$ Department of Applied Mathematics and Theoretical Physics, \\ University of Cambridge, Cambridge CB3 0WA, UK \\ ${ }^{2}$ BP Institute, University of Cambridge, Cambridge, CB3 0EZ, UK \\ ${ }^{3}$ Department of Earth Sciences, University of Cambridge, Cambridge CB3 0EZ, UK \\ ${ }^{4}$ Sichuan Energy Internet Research Institute, Tsinghua University, Chengdu, 610213, CN
}

(Received October 16, 2018; revised ?; accepted ?. - To be entered by editorial office)

We study the dynamics of two-phase flows injected into a confined porous layer. A model is derived to describe the evolution of the fluid-fluid interface, where the effective saturation of the injected fluid is zero, as the flow is driven by pressure gradients of injection, the buoyancy due to density contrasts and the interfacial tension between the injected and ambient fluids. The saturation field is then computed once the interface evolution is obtained. The results demonstrate that the flow behaviour evolves from early-time unconfined to late-time confined behaviours. In particular, at early times, the influence of capillary forces drive fluid flow and produce a new self-similar spreading behaviour in the unconfined limit, distinct from the gravity current solution. At late times, we obtain two new similarity solutions, a modified shock and a compound wave, in addition to the rarefaction and shock solutions in the sharp-interface limit. A schematic regime diagram is also provided, which summarizes all possible similarity solutions and the time transitions between them for the partially saturating flows resulting from fluid injection into a confined porous layer. Three dimensionless control parameters are identified and their influence on the fluid flow is also discussed, including the viscosity ratio, the pore-size distribution and the relative contributions of capillary and buoyancy forces. To underline the relevance of our results, we also briefly describe the implications of the two-phase flow model to the geological storage of $\mathrm{CO}_{2}$, using representative geological parameters from the Sleipner and In Salah sites.

Key words: gravity currents, multi-phase flow, porous media

\section{Introduction}

The flow of two fluid phases within a porous medium occurs in many environmental, geophysical and industrial processes including the motion of groundwater in porous aquifers (e.g., Bear 1972), the production of natural resources in subsurface reservoirs (e.g., Lake 1989), the storage of liquid waste in deep porous reservoirs, and the sequestration of $\mathrm{CO}_{2}$ in geological formations (e.g., Huppert \& Neufeld 2014). The flow behaviour can be complicated, given that it can be driven by forces including the background pressure gradient from fluid injection, buoyancy and capillary forces. It is of fundamental and practical interests to understand the role of these different driving forces at different time and length scales. 
Many previous studies focus on the case where a sharp, or distinct, interface can be identified between the injected and displaced fluids. Such a flow situation exists when the capillary forces and mixing between the two fluids are negligible. For example, previous research has been conducted to investigate the fluid motion and interface dynamics during fluid injection in both unconfined and confined porous media (e.g., Huppert \& Woods 1995; Lyle et al. 2005; Nordbotten \& Celia 2006; Pegler et al. 2014; Zheng et al. 2015a; Guo et al. 2016b). In addition, motivated by the application of geological $\mathrm{CO}_{2}$ sequestration, more recent work has focused on the effects of slow drainage or leakage systematically, including fluid drainage from a permeable caprock (e.g., Acton et al. 2001; Pritchard et al. 2001; Woods \& Farcas 2009; Zheng et al. 2015c; Liu et al. 2017), a finite edge (Hesse \& Woods 2010; Zheng et al. 2013; Yu et al. 2017), geological faults or leaky wells (e.g., Gasda et al. 2004; Neufeld et al. 2009, 2011; Vella et al. 2011)..

Capillary forces can significantly modify the behaviour of multi-phase flows in at least three ways. First, the immiscible fluids may each partially fill the pore space, hence the partial saturation must be tracked and an effective relative permeability determined which characterises the flow of one fluid past another (e.g., Buckley \& Leverett 1942; LeVeque 2002). Second, the capillary pressure jump between the injected and displaced fluids can also drive fluid flow, in flows driven by buoyancy and pressure gradients associated with fluid injection (e.g., de Gennes et al. 2004). Third, a fraction of the wetting phase can remain trapped within the solid matrix during fluid displacement, which results in an irreducible saturation of the wetting fluid (e.g., Hesse et al. 2008; Farcas \& Woods 2009; MacMinn et al. 2010).

A series of previous studies have considered the effects of residual trapping in a porous medium by assuming that a constant fraction of the wetting fluid is trapped during the fluid flow, which indicates a reduction in the effective porosity in the sharp-interface models. For example, a modified sharp-interface model has been proposed and a selfsimilar solution of the second kind is obtained to describe the dynamics of groundwater slumping in an aquifer with residual trapping at a constant rate (Kochina et al. 1983). In the context of geological $\mathrm{CO}_{2}$ storage, similar models have been proposed to describe how much and how fast is $\mathrm{CO}_{2}$ trapped after being injected into a saline aquifer (e.g., Hesse et al. 2008; Farcas \& Woods 2009; Juanes et al. 2010; MacMinn et al. 2010, 2011). However, these modified, sharp-interface models only consider a constant saturation and do not take into account the relative permeability experienced by each fluid phase, nor the possibility that the capillary pressure between phases may drive fluid flow.

To account more accurately for the effects of capillary forces, two-phase gravity current models have been developed for flows that partially saturates an unconfined porous medium, including the saturation-dependent capillary pressure, relative permeabilities and residual trapping (e.g., Gasda et al. 2009; Golding et al. 2011, 2013, 2017). Inspired by the practice of geological $\mathrm{CO}_{2}$ sequestration, these studies have focused on the steadystate flows generated from coupling fluid injection and edge drainage (Golding et al. 2011), radial spreading from vertical well injection (Golding et al. 2013), and horizontal propagation from an instantaneous release of a finite volume of fluid behind a lock gate (Golding et al. 2017). In all these studies a vertical capillary-gravity balance is assumed, and the time scale over which this balance is attained quantified (Golding et al. 2011; Nordbotten \& Dahle 2011). The influence of confinement on the dynamical evolution has only been examined chiefly for sharp-interface, single phase (i.e., immiscible) currents. These studies identified a transition from an early-time, unconfined self-similar behaviour to three different branches of late-time confined self-similar behaviours, depending on the viscosity ratio of the two fluids (Pegler et al. 2014; Zheng et al. 2015a). Numerical models of two-phase flows in confined layers have also recently been formulated, computing either 
the mean saturation (integrated over the reservoir depth, Nordbotten \& Dahle 2011) or the the two-phase, fluid-fluid interface (Nilsen et al. 2016). These studies demonstrated the utility of the confined, two-phase formulation, by focusing on model development illustrated by industrially-relevant case studies.

Here we focus instead on the dynamical regimes present during the injection of a twophase flow into a confined porous layer. In this paper, we first describe a theoretical model in $\S 2$ for two phase flows due to fluid injection into a confined porous layer. Then, in $\S 3$, we provide an example calculation, employing a specific set of capillary pressure and relative permeability curves taken from a geological $\mathrm{CO}_{2}$ sequestration project, and derive the early-time and late-time self-similar asymptotic solutions for the evolution of the interface shape. In $\S 4$, we perform a detailed numerical calculation for the governing partial differential equation, and compare the results of direct numerical simulations with the self-similar solutions we derived in $\S 3$ in various asymptotic limits. A schematic regime diagram is provided in $\S 5$, which summarizes the dynamic evolution of the partially saturating flows; the influence of different control parameters on the self-similar solutions in the regime schematic is also addressed. Finally, in $\S 6$ we briefly discuss the possible implications of the current model to the geological $\mathrm{CO}_{2}$ sequestration projects, employing representative geological parameters from the Sleipner and In Salah sites.

\section{Theoretical model}

\subsection{Two-phase flows in porous media}

We consider a two-phase flow of non-wetting fluid injected into a homogeneous and isotropic porous medium of porosity $\phi$ and permeability $k$, initially fully saturated by a wetting fluid. The volume fraction of the non-wetting and wetting fluids in a representative elementary volume $(\mathrm{REV})$ is $\phi_{n}$ and $\phi_{w}$, respectively, while the saturation of the two fluids is

$$
S_{n}=\phi_{n} / \phi \quad \text { and } \quad S_{w}=\phi_{w} / \phi .
$$

Treating the flow of both fluids and the solid matrix as incompressible, mass conservation within the pore space therefore dictates that

$$
S_{n}+S_{w}=1 \text {. }
$$

Because of capillary effects, there is often an irreducible fraction (or saturation) of the wetting fluid left in the porous medium, $S_{w i}$. We define the effective non-wetting phase saturation and effective wetting phase saturation as

$$
s \equiv \frac{S_{n}}{1-S_{w i}} \quad \text { and } \quad 1-s=\frac{S_{w}-S_{w i}}{1-S_{w i}}
$$

respectively, corresponding to the empirical behaviours of partially saturating flows (e.g., Leverett 1941; Brooks \& Corey 1964; Bennion \& Bachu 2005). We note that in general, the effective non-wetting saturation $s(\boldsymbol{x}, t)$ depends on space $\boldsymbol{x}$ and time $t$.

We use standard empirical models for the capillary pressure, $p_{c}$, which relates the pressure in the nonwetting and wetting fluid phases, $p_{n}$ and $p_{w}$, to the local saturation,

$$
p_{n}-p_{w}=p_{c}(s) \text {. }
$$

Here we use the Brooks-Corey model (Brooks \& Corey 1964) which assumes a particularly convenient power-law form

$$
p_{c}(s)=p_{e}(1-s)^{-1 / \Lambda} \text {, }
$$



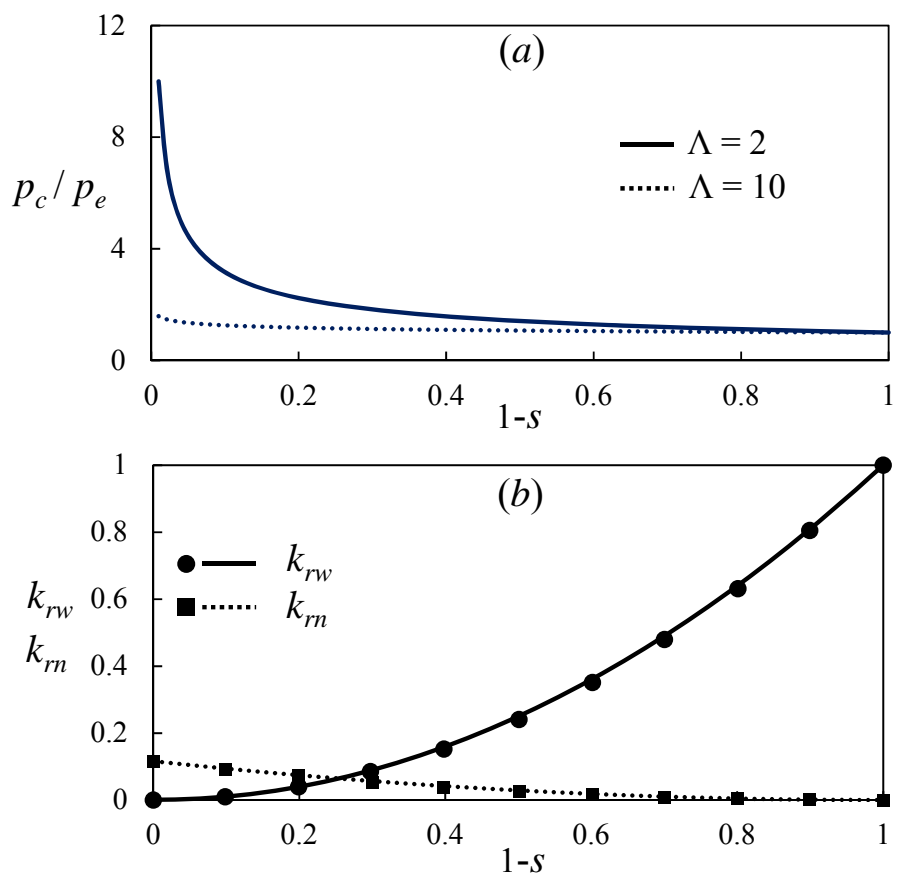

Figure 1. Capillary pressure in $(a)$ and relative permeability curves in $(b)$. The symbols in $(b)$ are representative values of relative permeabilities taken from a $\mathrm{CO}_{2}$ geological sequestration projects (e.g., Bennion \& Bachu 2005; Li \& Horne 2006), and the curves represent best power-law fitting results in (2.7) with $k_{r n 0}=0.116$ and $\alpha=\beta=2$ (Bennion \& Bachu 2005; Golding et al. 2011).

where $p_{e}$ is the capillary entry pressure, and $\Lambda$ is a fitting parameter that characterizes the pore-size distribution of the porous medium. Smaller values of $\Lambda$ correspond to a wider distribution of pore sizes of the porous medium, and $\Lambda \rightarrow \infty$ is the limiting case of monodisperse pores, as shown in figure $1 a$.

To compute the volumetric flux for the non-wetting $\left(\boldsymbol{u}_{\boldsymbol{n}}\right)$ and wetting $\left(\boldsymbol{u}_{\boldsymbol{w}}\right)$ phases, we use a standard multiphase extension of Darcy's law (e.g., Leverett 1941; Bear 1972; Phillips 1991)

$$
\begin{aligned}
& \boldsymbol{u}_{n}=-\frac{k k_{r n}}{\mu_{n}}\left(\nabla p_{n}-\rho_{n} \boldsymbol{g}\right), \\
& \boldsymbol{u}_{w}=-\frac{k k_{r w}}{\mu_{w}}\left(\nabla p_{w}-\rho_{w} \boldsymbol{g}\right),
\end{aligned}
$$

where $\mu_{n}$ and $\mu_{w}$ are the viscosity of the non-wetting and wetting fluids respectively, and $k_{r n}(s)$ and $k_{r w}(s)$ are the (dimensionless) relative permeabilities of the non-wetting and wetting phases, which we assume to be solely a function of the saturation,

$$
\begin{aligned}
& k_{r n}(s)=k_{r n 0} s^{\alpha}, \\
& k_{r w}(s)=(1-s)^{\beta} .
\end{aligned}
$$

Here $k_{r n 0}$ is the end-point relative permeability of the non-wetting phase, and $\alpha$ and $\beta$ are fitting parameters (e.g., Bennion \& Bachu 2005; Li \& Horne 2006; Golding et al. 2011, 2013). A representative set of values, applied previously in the context of geological $\mathrm{CO}_{2}$ 

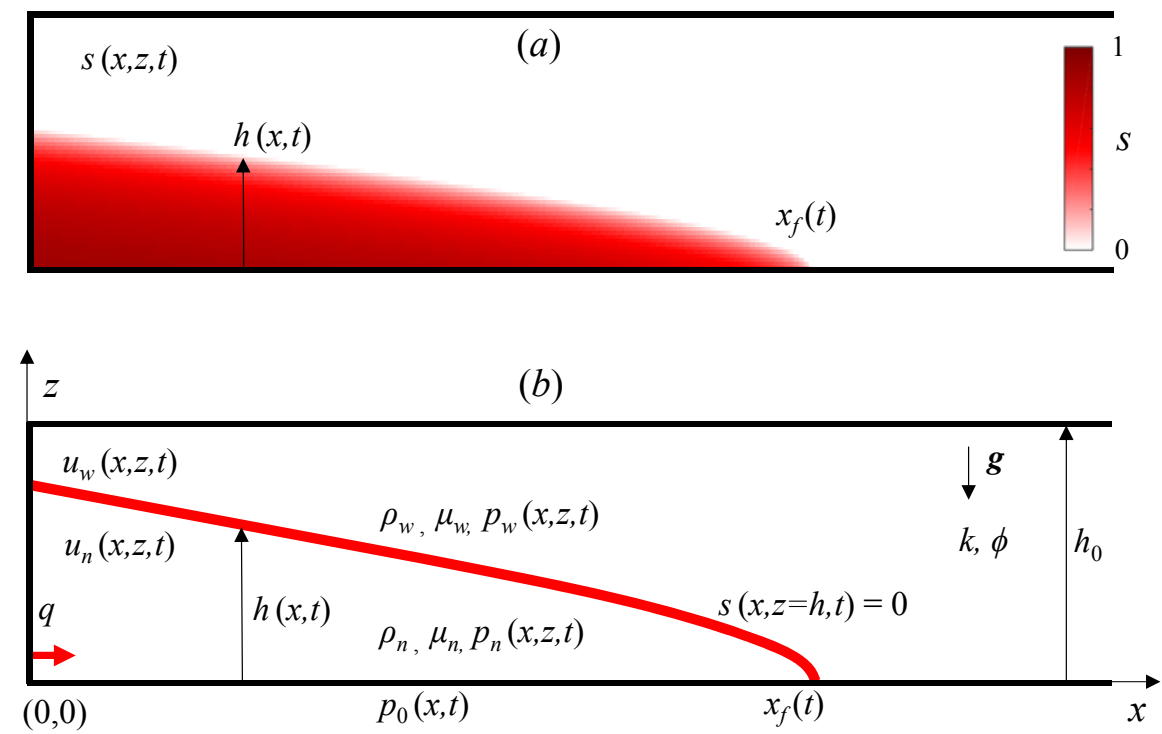

FIGURE 2. Schematic of the injection of a non-wetting fluid into a confined porous layer initially saturated with a wetting fluid: $(a)$ shows the saturation field of the injected fluid; $(b)$ illustrates that the interface $h(x, t)$ is defined as the location where the effective saturation of the injected non-wetting fluid $s(x, z, t)=0$, and $x_{f}(t)$ denotes the location of the propagating front.

sequestration, is $k_{r n 0}=0.116$ and $\alpha=\beta=2$ (Bennion \& Bachu 2005; Golding et al. 2011), as shown in figure $1 b$.

\subsection{Confined, two-phase gravity currents}

We now consider the propagation of a two-phase gravity current in a confined homogeneous porous layer of constant and uniform porosity $\phi$, intrinsic permeability $k$, and bounded by impermeable horizontal boundaries at $z=0$ and $h_{0}$, as shown in figure 2 . A non-wetting fluid of density $\rho_{n}$ is injected at $(x, z)=(0,0)$, and displaces the wetting fluid of density $\rho_{w}$ (both fluid phases are assumed incompressible). Without loss of generality, we assume that the injected fluid is more dense than the displaced fluid, i.e., $\Delta \rho=\rho_{n}-\rho_{w}>0$, but note that the dynamics are identical for $\Delta \rho<0$ when the current propagates along the top of the confined layer. The interface is located at $z=h(x, t)$, where the effective saturation of the non-wetting phase $s$ becomes zero, and is a function of the horizontal coordinate $x$ and time $t$. According to (2.5), the pressure jump at the interface is the capillary entry pressure $p_{e}$.

We assume that the current is long and thin, and hence the flow is mainly horizontal, and the pressure in both phases is approximately hydrostatic,

$$
\begin{aligned}
& p_{n}(x, z, t)=p_{0}(x, t)-\rho_{n} g z, \quad 0 \leqslant z \leqslant h(x, t), \\
& p_{w}(x, z, t)=p_{0}(x, t)-\rho_{n} g h(x, t)-\rho_{w} g[z-h(x, t)]-p_{e}, \quad 0 \leqslant z \leqslant h_{0},
\end{aligned}
$$

where $p_{0}(x, t)$ is the pressure distribution of the injected fluid along the bottom boundary. We also note that, compared with the sharp interface models described in Pegler et al. (2014) and Zheng et al. (2015a), the capillary entry pressure, $p_{e}$, now appears in the pressure distribution $(2.8 b)$, which represents the pressure jump due to capillary effects at the fluid-fluid interface $h(x, t)$. The saturation may therefore be inferred from (2.4), (2.5) and (2.8) in a manner consistent with the gravity-capillary balance detailed previously 
(the speed at which gravity-capillary equilibrium is reached is rapid for high aspect ratio currents, see, e.g., Golding et al. 2011; Nordbotten \& Dahle 2011),

$$
s= \begin{cases}1-\left(1+\frac{h-z}{h_{e}}\right)^{-\Lambda}, & 0 \leqslant z \leqslant h(x, t), \\ 0, & h(x, t) \leqslant z \leqslant h_{0},\end{cases}
$$

where $h_{e} \equiv p_{e} / \Delta \rho g$ is the characteristic height of the capillary fringe. We note that $s(x, z, t)=s[h(x, t), z]$, so that the dependence of the saturation on $x$ and $t$ is now included in the information of the interface shape $h(x, t)$.

The horizontal velocities within the non-wetting and wetting phases are

$$
\begin{aligned}
& u_{n}(x, z, t)=-\frac{k k_{r n}(s)}{\mu_{n}} \frac{\partial p_{n}(x, z, t)}{\partial x}, \\
& u_{w}(x, z, t)=-\frac{k k_{r w}(s)}{\mu_{w}} \frac{\partial p_{w}(x, z, t)}{\partial x},
\end{aligned}
$$

respectively, where we assume that the relative permeability functions $k_{r n}(s)$ and $k_{r w}(s)$ depend only on the saturation field $s=s[h(x, t), z]$, given by $(2.9)$.

In addition, the non-wetting fluid is injected at a constant volumetric rate $q$, and hence at each location mass conservation requires

$$
q=\int_{0}^{h(x, t)} u_{n}(x, z, t) \mathrm{d} z+\int_{0}^{h_{0}} u_{w}(x, z, t) \mathrm{d} z,
$$

where we note that the non-wetting phase only exists between $0 \leqslant z \leqslant h(x, t)$, while the wetting phase occupies the entire layer $0 \leqslant z \leqslant h_{0}$. This local mass conservation may be used to infer the background pressure gradient $\partial p_{0} / \partial x$. Substituting (2.8) into (2.10), and then (2.10) into (2.11), we obtain

$$
\frac{\partial p_{0}}{\partial x}(x, t)=\frac{\Delta \rho g I_{w}(h)}{M I_{n}(h)+I_{w}(h)} \frac{\partial h}{\partial x}-\frac{q \mu_{w} / k}{M I_{n}(h)+I_{w}(h)},
$$

where $M \equiv \mu_{w} / \mu_{n}$ is the viscosity ratio of the displaced (wetting) fluid over the injected (non-wetting) fluid. Here $I_{w}(h)$ and $I_{n}(h)$ are the vertically integrated relative permeability functions, defined as

$$
\begin{aligned}
I_{w}(h) & \equiv \int_{0}^{h_{0}} k_{r w}(s) \mathrm{d} z, \\
I_{n}(h) & \equiv \int_{0}^{h(x, t)} k_{r n}(s) \mathrm{d} z,
\end{aligned}
$$

and the saturation function $s[h(x, t), z]$ is provided by (2.9). By substituting (2.12) into $(2.10 a, b)$, the velocity fields $u_{n}(x, z, t)$ and $u_{w}(x, z, t)$ can be computed as the interface $h(x, t)$ evolves.

Local continuity of the injected non-wetting fluid states that

$$
\frac{\partial}{\partial t} \int_{0}^{h(x, t)} \phi_{n}(x, z, t) \mathrm{d} z+\frac{\partial}{\partial x} \int_{0}^{h(x, t)} u_{n}(x, z, t) \mathrm{d} z=0 .
$$

We note that $\phi_{n}(x, z, t)=\phi\left(1-S_{w i}\right) s[h(x, t), z]$, and we define the vertically-integrated saturation function as

$$
I_{s}(h) \equiv \int_{0}^{h(x, t)} s[h(x, t), z] \mathrm{d} z=h+\frac{h_{e}}{1-\Lambda}\left[1-\left(1+\frac{h}{h_{e}}\right)^{1-\Lambda}\right],
$$


where we have used the expression (2.9) for the effective saturation $s[h(x, t), z]$. Using (2.15), $(2.10 a)$ and (2.14), we obtain the evolution equation for the interface shape $h(x, t)$ for a two-phase gravity current in a confined porous layer,

$$
\phi\left(1-S_{w i}\right) \frac{\partial I_{s}(h)}{\partial t}+q \frac{\partial}{\partial x}\left[\frac{M I_{n}(h)}{M I_{n}(h)+I_{w}(h)}\right]-\frac{\Delta \rho g k}{\mu_{n}} \frac{\partial}{\partial x}\left[\frac{I_{n}(h) I_{w}(h)}{M I_{n}(h)+I_{w}(h)} \frac{\partial h}{\partial x}\right]=0,
$$

where the integrated saturations are given by $(2.13 a, b)$ and $(2.15)$. We provide the appropriate initial and boundary conditions in $\S 2.2 .1$ to complete the problem.

\subsubsection{Boundary conditions and the initial fluid distribution}

We assume that the medium is initially completely saturated with ambient fluid and that injection starts at time $t=0$. Thus, initially the saturation $s(x, 0)=0$ and so

$$
h(x, 0)=0 .
$$

At all times we define the front of the current by

$$
h\left[x_{f}(t), t\right]=0 .
$$

In addition, we assume that there is no flux through the nose of the current,

$$
\left.I_{n}(h) \frac{\partial h}{\partial x}\right|_{x=x_{f}(t)}=0 .
$$

Equation (2.18) is used to determine $x_{f}(t)$, given that $h(+\infty, t)=0$. A global statement of conservation of injected fluid gives

$$
\phi\left(1-S_{w i}\right) \int_{0}^{x_{f}(t)} I_{s}(h) \mathrm{d} x=q t,
$$

which, using (2.16) and (2.18), may be reformulated in terms of the flux of non-wetting fluid at the origin,

$$
\left.\left[\frac{q M I_{n}(h)}{M I_{n}(h)+I_{w}(h)}-\frac{\Delta \rho g k}{\mu_{n}} \frac{I_{n}(h) I_{w}(h)}{M I_{n}(h)+I_{w}(h)} \frac{\partial h}{\partial x}\right]\right|_{0}=q .
$$

Note that we have assumed that there is no-entrainment of ambient fluid (2.19), which has also been employed to derive the sharp-interface models (e.g., Zheng et al. 2015a).

The evolution equation, (2.16), is subject to the initial condition (2.17) and boundary conditions (2.18) and (2.21). Given the relative permeability functions $k_{n}(s)$ and $k_{w}(s)$, the integrals $I_{n}(h)$ and $I_{w}(h)$ can be evaluated according to (2.13), and the corresponding revised form of the evolution equation (2.16) can be obtained. Analytical and numerical tools can then be employed to solve for the evolution of the interface shape, $h(x, t)$, and the saturation distribution, $s[h(x, t), z]$, using $(2.9)$.

\subsection{Limiting behaviours of the evolution equation}

The evolution equation, (2.16), contains two main components: an advective term that describes flow driven by the pressure gradient due to fluid injection, and a diffusive term describing flows driven by the density difference (buoyancy) between the injected and ambient fluids. Equation (2.16) represents the multiphase extension of previous work on immiscible systems (e.g., Pegler et al. 2014; Zheng et al. 2015a) and is comparable to previous two-phase studies (Golding et al. 2011; Nordbotten \& Dahle 2011; Nilsen et al. 2016). Here we briefly describe how (2.16) recovers limits considered previously. We then detail new dynamical regimes from the multiphase formulation in $\S 3$ and discuss the 
time transition between regimes in $\S 4$. Specifically, we show that the evolution equation, (2.16), recovers the sharp-interface limit $(\S 2.3 .1)$, the unconfined flow limit $(\S 2.3 .2)$ and the confined flow limit $(\S 2.3 .3)$.

\subsubsection{The sharp-interface limit}

We first consider the limit when a sharp interface exists between the injected and displaced fluids. This limit is recovered in monodisperse porous media, $\Lambda \rightarrow \infty$, where no capillary fringe exists. In this limit, the saturation function $s[h(x, t), z]$ satisfies

$$
s[h(x, t), z]= \begin{cases}1, & 0 \leqslant z \leqslant h(x, t) \\ 0, & h(x, t) \leqslant z \leqslant h_{0}\end{cases}
$$

Thus, the integrals $I_{s}, I_{n}$, and $I_{w}$ can be computed as

$$
I_{s}=h, I_{n}=k_{r n 0} h, \text { and } I_{w}=h_{0}-h,
$$

leading to a reduced, sharp-interface model

$$
\phi\left(1-S_{w i}\right) \frac{\partial h}{\partial t}+q \frac{\partial}{\partial x}\left[\frac{M k_{r n 0} h}{\left(M k_{r n 0}-1\right) h+h_{0}}\right]-\frac{\Delta \rho g k}{\mu_{n}} \frac{\partial}{\partial x}\left[\frac{k_{r n 0} h\left(h_{0}-h\right)}{\left(M k_{r n 0}-1\right) h+h_{0}} \frac{\partial h}{\partial x}\right]=0 .
$$

Equation (2.24) effectively recovers an analogous form of the evolution equation for sharpinterface gravity currents propagating in a confined porous layer, i.e., equation (2.6) in Zheng et al. (2015a), or equation (3.6) in Pegler et al. (2014). The only difference is the inclusion of the effects of the irreducible wetting phase saturation $S_{w i}$, and the endpoint relative permeability of the non-wetting phase, $k_{r n 0}$. By setting the two constants $S_{w i}=0$ and $k_{r n 0}=1,(2.24)$ exactly recovers those previous descriptions of immiscible confined gravity currents.

\subsubsection{The limit of effectively unconfined flow}

At early times, when $h \ll h_{0}$, the flow is effectively unconfined and the pressure gradients associated with fluid injection are much smaller than that due to buoyancy. In addition, $\left|M I_{n}(h)\right| \ll\left|I_{w}(h)\right|$, which reduces to $M k_{r n 0} h \ll h_{0}$ in the sharp-interface limit. Equation (2.16) then reduces to

$$
\phi\left(1-S_{w i}\right) \frac{\partial I_{s}(h)}{\partial t}-\frac{\Delta \rho g k}{\mu_{n}} \frac{\partial}{\partial x}\left[I_{n}(h) \frac{\partial h}{\partial x}\right]=0,
$$

which is the governing equation for unconfined gravity currents, i.e. equation (3.8) in Golding et al. (2011). We provide a more detailed discussion in $§ 3.3$.

\subsubsection{The limit of effectively confined flow}

When the pressure gradient associated with injection is much greater than the hydrostatic pressure gradient, (2.16) is purely advective and reduces to

$$
\phi\left(1-S_{w i}\right) \frac{\partial I_{s}(h)}{\partial t}+q \frac{\partial}{\partial x}\left[\frac{M I_{n}(h)}{M I_{n}(h)+I_{w}(h)}\right]=0,
$$

which recovers the form of the Buckley-Leverett equation for two-phase flows in confined porous media (e.g., Buckley \& Leverett 1942; LeVeque 2002). We also note that the Buckley-Leverett equation was derived in the limit of zero capillary effects (Buckley \& Leverett 1942), while (2.26) includes an effective parameterisation of capillary effects. In $\S 3.4$ we show that, assuming the effects of buoyancy-driven flow (diffusion term) are negligible, this approximate holds at late times when the flow is confined. We also 


\author{
Comments \\ modified viscosity ratio \\ rescaled capillary length \\ pore size distribution
}

TABLE 1. Three dimensionless control parameters are identified: the modified viscosity ratio $N$, rescaled capillary length $H_{e}$ and pore size distribution parameter $\Lambda$.

note that recent studies (e.g. Pegler et al. 2014; Zheng et al. 2015a) provide detailed calculations for the effects of the buoyancy term in the sharp interface limit.

\section{Example calculations}

To provide concrete examples of the behaviour of confined, two-phase flows we use representative, power-law relative permeability functions $k_{n}(s)$ and $k_{w}(s),(2.7 a, b)$, and evaluate the integrals $I_{n}(h)$ and $I_{w}(h)$ according to $(2.13)$. With this choice we study the early-time and late-time asymptotic behaviours during the evolution of the interface shape $h(x, t)$, as described by (2.16). However, we note that the theoretical framework could be readily applied to other flow situations with alternate forms of the capillary pressure and relative-permeability functions.

\subsection{Revised evolution equation}

Here we take $\alpha=2$ and $\beta=2$, motivated by the experimental data from the Ellerslie standstone system (Bennion \& Bachu 2005), and also assume $\Lambda \neq 1,1 / 2$. Using (A 1), (A 3) and (2.16) we obtain the revised form of the evolution equation

$$
\phi\left(1-S_{w i}\right) f_{s}(h) \frac{\partial h}{\partial t}+q \frac{\partial}{\partial x}\left[\frac{M f_{n}(h)}{M f_{n}(h)+f_{w}(h)}\right]-\frac{\Delta \rho g k}{\mu_{n}} \frac{\partial}{\partial x}\left[\frac{f_{n}(h) f_{w}(h)}{M f_{n}(h)+f_{w}(h)} \frac{\partial h}{\partial x}\right]=0,
$$

where

$$
\begin{aligned}
f_{s}(h) & \equiv 1-\left(1+\frac{h}{h_{e}}\right)^{-\Lambda} \\
f_{n}(h) & \equiv k_{r n 0}\left(h+\frac{2 h_{e}}{1-\Lambda}\left[1-\left(1+\frac{h}{h_{e}}\right)^{1-\Lambda}\right]-\frac{h_{e}}{1-2 \Lambda}\left[1-\left(1+\frac{h}{h_{e}}\right)^{1-2 \Lambda}\right]\right) \\
f_{w}(h) & \equiv\left(h_{0}-h\right)+\frac{h_{e}}{1-2 \Lambda}\left[1-\left(1+\frac{h}{h_{e}}\right)^{1-2 \Lambda}\right] .
\end{aligned}
$$

We note that, $I_{n}(h)$ and $I_{w}(h)$, in particular, can be evaluated explicitly for special values of $\alpha$ (appendix A). We study equation (3.1) in this paper, as a representative example, to demonstrate the dynamics inherent in solutions of the two-phase gravity current model, incorporating capillary effects. 


\subsection{Non-dimensionalization}

We now nondimensionalize the evolution equation, (3.1), and its initial and boundary conditions, $(2.17),(2.18)$ and $(2.21)$, by choosing appropriate time and length scales. The natural vertical scale is the thickness of the porous layer, $h_{0}$. We define dimensionless variables $H \equiv h / h_{c}, X \equiv x / x_{c}$, and $T \equiv t / t_{c}$, where

$$
h_{c}=h_{0}, \quad x_{c}=\frac{\Delta \rho g k k_{r n 0} h_{0}^{2}}{\mu_{n} q}, \quad t_{c}=\frac{\Delta \rho g k k_{r n 0} \phi\left(1-S_{w i}\right) h_{0}^{3}}{\mu_{n} q^{2}},
$$

are the characteristic length and time scales, respectively. We note that $x_{c}$ and $t_{c}$ are chosen such that $T \sim 1$ indicates the time scale when both injection and buoyancy effects are equally important in driving the fluid flow. In this way, we obtain the dimensionless governing equation for the interface shape $H(X, T)$

$$
\mathcal{F}_{s}(H) \frac{\partial H}{\partial T}+\frac{\partial}{\partial X}\left[\frac{N \mathcal{F}_{n}(H)}{N \mathcal{F}_{n}(H)+\mathcal{F}_{w}(H)}\right]-\frac{\partial}{\partial X}\left[\frac{\mathcal{F}_{n}(H) \mathcal{F}_{w}(H)}{N \mathcal{F}_{n}(H)+\mathcal{F}_{w}(H)} \frac{\partial H}{\partial X}\right]=0,
$$

where

$$
\begin{aligned}
& \mathcal{F}_{s}(H) \equiv 1-\left(1+\frac{H}{H_{e}}\right)^{-\Lambda} \\
& \mathcal{F}_{n}(H) \equiv H+\frac{2 H_{e}}{1-\Lambda}\left[1-\left(1+\frac{H}{H_{e}}\right)^{1-\Lambda}\right]-\frac{H_{e}}{1-2 \Lambda}\left[1-\left(1+\frac{H}{H_{e}}\right)^{1-2 \Lambda}\right] \\
& \mathcal{F}_{w}(H) \equiv(1-H)+\frac{H_{e}}{1-2 \Lambda}\left[1-\left(1+\frac{H}{H_{e}}\right)^{1-2 \Lambda}\right]
\end{aligned}
$$

Two new dimensionless parameters are defined in equation (3.4) that govern the behaviour of the propagating current

$$
N \equiv k_{r n o} \mu_{w} / \mu_{n}, \text { and } H_{e} \equiv h_{e} / h_{0} .
$$

Thus, there are, in total, three dimensionless parameters in the problem: $N, H_{e}, \Lambda$, as summarized in table 1 . Here $N$ is a modified viscosity ratio, which is analogous to $M$, the viscosity ratio in the sharp-interface model (e.g., Pegler et al. 2014; Zheng et al. $2015 a) . H_{e}$ measures the strength of the capillary over buoyancy forces, and $\Lambda$, as first introduced in $\S 2.1$, characterises the distribution of pore sizes in the porous medium. We note that the unconfined two-phase gravity current model (e.g., Golding et al. 2011, 2013) only includes two dimensionless parameters $H_{e}$ and $\Lambda$. Here, where confinement is important, the parameter $N$ describes the pressure gradient needed to displace the ambient (wetting) fluid when the thickness of the interface shape is comparable with the thickness of the porous layer.

In addition, the dimensionless initial and boundary conditions become

$$
H(X, 0)=0,
$$

$$
H\left[X_{f}(T), T\right]=0
$$

and, at the origin

$$
\left.\left[\frac{N \mathcal{F}_{n}(H)}{N \mathcal{F}_{n}(H)+\mathcal{F}_{w}(H)}-\frac{\mathcal{F}_{n}(H) \mathcal{F}_{w}(H)}{N \mathcal{F}_{n}(H)+\mathcal{F}_{w}(H)} \frac{\partial H}{\partial X}\right]\right|_{X=0}=1 .
$$

Now the dimensionless governing equation, (3.4), can be solved numerically, subject to 

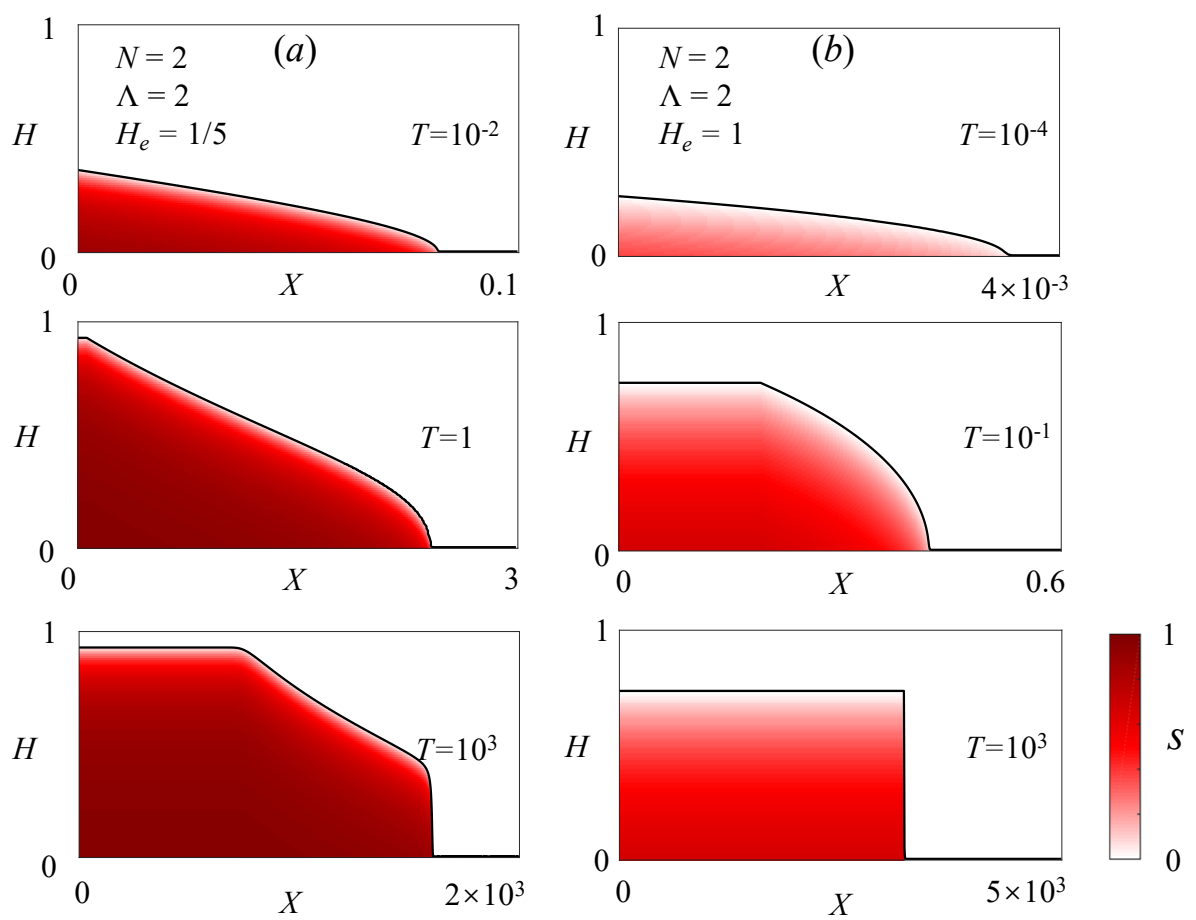

FiguRE 3. Representative calculations for the evolution of the profile shape (black curves) and the saturation field: $(a)$ for $N=2, \Lambda=2, H_{e}=1 / 5$ and $(b)$ for $N=2, \Lambda=2, H_{e}=1$. The evolution of the interface shape $H(X, T)$, obtained from numerical solutions of PDE (3.4), indicates a transition from early-time unconfined to late-time confined flow behaviours. Once the interface shape is obtained, the saturation field is calculated based on (3.10).

initial condition (3.7) and boundary conditions (3.8) and (3.9), to provide the solution for the evolution of the interface shape $H(X, T)$. Representative numerical results for $H(X, T)$ at different times are shown in figure 3 .

Once the solution for the interface shape $H(X, T)$ is obtained, based on $(2.9)$, in the dimensionless coordinates $(X, Z)$ with $Z \equiv z / h_{0}$, the saturation distribution $s[H(X, T), Z]$ can also be computed according to

$$
s[H(X, T), Z]= \begin{cases}1-\left(1+\frac{H-Z}{H_{e}}\right)^{-\Lambda}, & 0 \leqslant Z \leqslant H(X, T), \\ 0, & H(X, T) \leqslant Z \leqslant 1 .\end{cases}
$$

Representative results of $s[H(X, T), Z]$ based on the numerical solutions of (3.4) subject to (3.7)-(3.9) are shown in figure 3 , which demonstrates the effects of capillary forces on the propagation of a gravity current in a porous medium. In particular, compared with the prediction of the sharp-interface model, the saturation of the injected non-wetting fluid in figure 3 varies in time and space continuously, due to the existence of a capillary fringe. As a result, the location of the propagating front and the interface shape, defined as where the saturation for the injected fluid is zero, can be different from the prediction of the sharp-interface model in previous studies (e.g., Pegler et al. 2014; Zheng et al. 2015a). In addition, the value of $\Lambda$ and $H_{e}$ indicates the strength of the capillary effects, and we show the influence of $\Lambda$ and $H_{e}$ in figure 4, where the saturation field approaches the sharp-interface limit as $\Lambda \rightarrow \infty$ and $H_{e} \rightarrow 0^{+}$. 
(a)

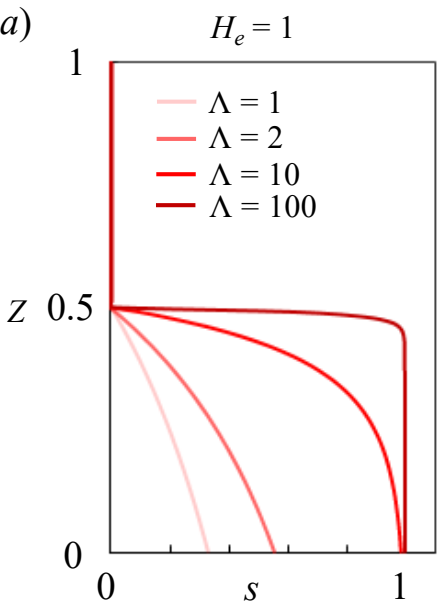

(b)

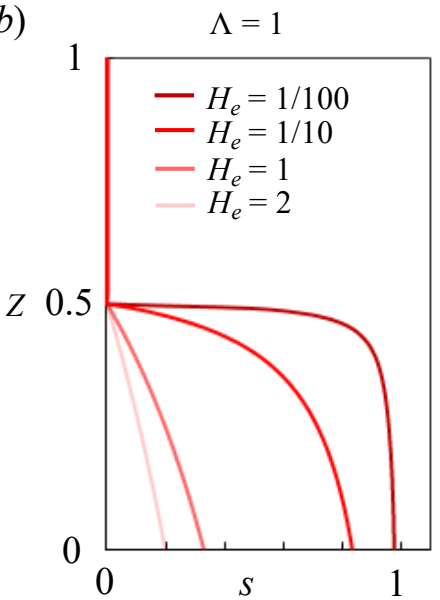

FiguRE 4. Influence of $\Lambda$ and $H_{e}$ on the saturation field based on (3.10) with $H=1 / 2$ as an example. (a) $H_{e}=1$ and $\Lambda=\{1,2,10,100\}$ and $(b)$ for $\Lambda=1$ and $H_{e}=\{1 / 100,1 / 10,1,2\}$. As $H_{e} \rightarrow \infty$ or $\Lambda \rightarrow 0^{+}$, the saturation field approaches the sharp-interface limit.

The form of (3.4) suggests that, at $T=\mathcal{O}(1)$, both the advective (injection) and diffusive (buoyancy) terms are important for the interface shape $H(X, T)$. However, for early or late times, the advective and diffusive terms have different orders of magnitude, which motivates us to look for the different asymptotic behaviours in $\S 3.3$ and $\S 3.4$ and investigate, in different asymptotic limits, the difference between the prediction of the sharp-interface model and the current model of two-phase partially saturating flow.

\subsection{Early-time asymptotic solutions}

At early times, $T \ll 1$, the length of the current $X \ll 1$ and the thickness $H \ll 1$, and the flow is effectively unconfined. Flow of the ambient is negligible and the pressure gradient associated with injection may be neglected, which we justify a posteriori. In this limit, we recover the model for a two-phase gravity current spreading in an unconfined porous medium (e.g., Golding et al. 2011, 2013, 2017)

$$
\mathcal{F}_{s}(H) \frac{\partial H}{\partial T}-\frac{\partial}{\partial X}\left[\mathcal{F}_{w}(H) \frac{\partial H}{\partial X}\right]=0
$$

The dimensionless statement of global mass conservation may now be written as

$$
\int_{0}^{X_{f}(T)} \int_{0}^{H}\left[1-\left(1+\frac{H-Z}{H_{e}}\right)^{-\Lambda}\right] \mathrm{d} Z \mathrm{~d} X=T
$$

which determines the front location $X_{f}(T)$.

The model includes the dimensionless parameter $H_{e}$, which measures the strength of the capillary forces. Note that the thickness $H$ increases as injection continues, and hence there is a crossover time when the height of the current is comparable to the capillary height, $H \sim H_{e}$, assuming that the capillary length is smaller than the thickness of the porous medium, $H_{e}<1$. We can further explore two distinct limits at early times in the asymptotic behaviours for the unconfined two-phase flow. When $H \ll H_{e}$, the capillary effects are initially dominant, and when $H \gg H_{e}$, buoyancy dominates over capillarity. For $H_{e} \gg 1$, capillary forces remain dominant throughout the evolution of the current. 

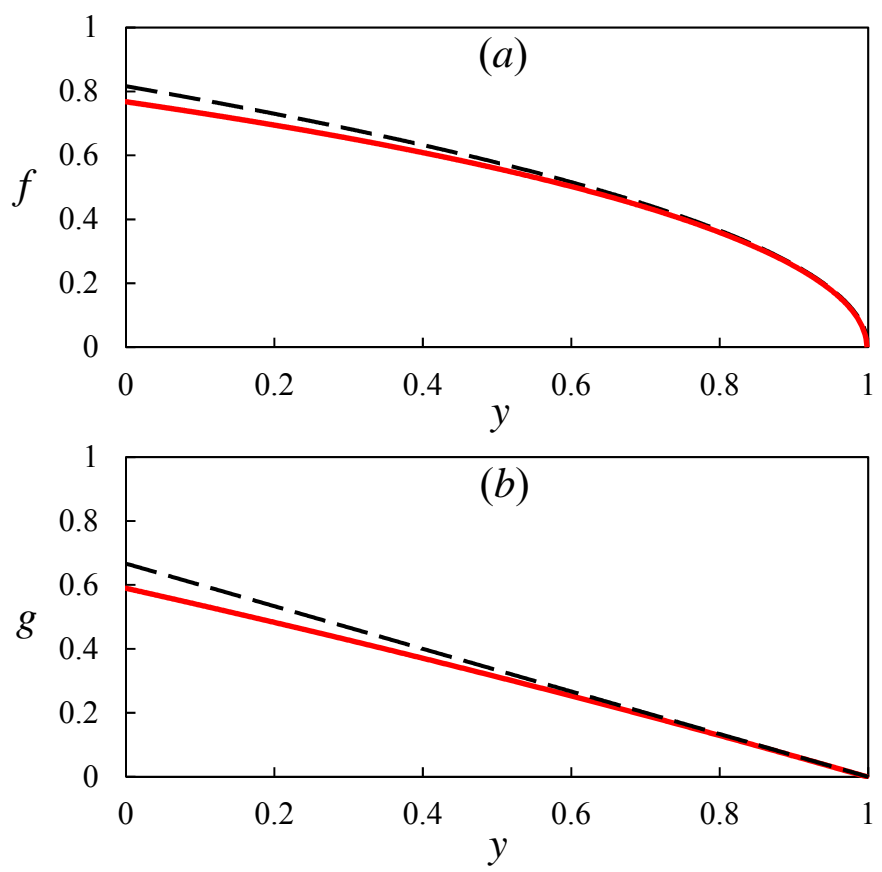

FIGURE 5. Early-time self-similar solutions: $(a)$ strong capillary regime (§3.3.1) and $(b)$ weak capillary regime (§3.3.2). The solid curves represent the numerical calculations of the similarity solutions. The dashed curves represent the asymptotic shapes near the front of the two-phase gravity current, i.e., solution (3.16) in $(a)$ and (3.23) in $(b)$.

\subsubsection{Strong capillarity regime: $H \ll H_{e}$}

Initially, as fluid is injected into the porous medium, $H \ll H_{e}$ and the capillary effects are strong. In this regime, (3.11) reduces to

$$
H \frac{\partial H}{\partial T}-\frac{\Lambda}{3 H_{e}} \frac{\partial}{\partial X}\left(H^{3} \frac{\partial H}{\partial X}\right)=0
$$

In addition, global mass conservation, (3.12), reduces to

$$
\frac{\Lambda}{H_{e}} \int_{0}^{X_{f}(T)} H^{2} \mathrm{~d} X=T
$$

This new regime, in which the flow is driven by capillary forces, has not previously been reported. A scaling argument suggests that in this limit $X \propto T^{2 / 3}$ and $H \propto T^{1 / 6}$.

With this motivation, we define a similarity variable $\xi \equiv 3^{1 / 3} X / T^{2 / 3}$, which suggests that the front propagates as $X_{f}(T)=\xi_{f} 3^{-1 / 3} T^{2 / 3}$, where $\xi_{f}$ is a constant to be determined. We normalize the self-similar length $y \equiv X / X_{f}(T)=\xi / \xi_{f}$ and write the interface shape as $H(X, T)=\xi_{f} 3^{1 / 6}\left(H_{e} / \Lambda\right)^{1 / 2} T^{1 / 6} f(y)$. Then, the shape $f(y)$ and the stretching constant $\xi_{f}$ can be determined by solving the following system of equations

$$
\begin{array}{r}
\left(f^{3} f^{\prime}\right)^{\prime}+\frac{2}{3} y f f^{\prime}-\frac{1}{6} f^{2}=0, \\
f(1)=0, \\
\xi_{f}=\left[\int_{0}^{1} f(y)^{2} \mathrm{~d} y\right]^{-1 / 3},
\end{array}
$$



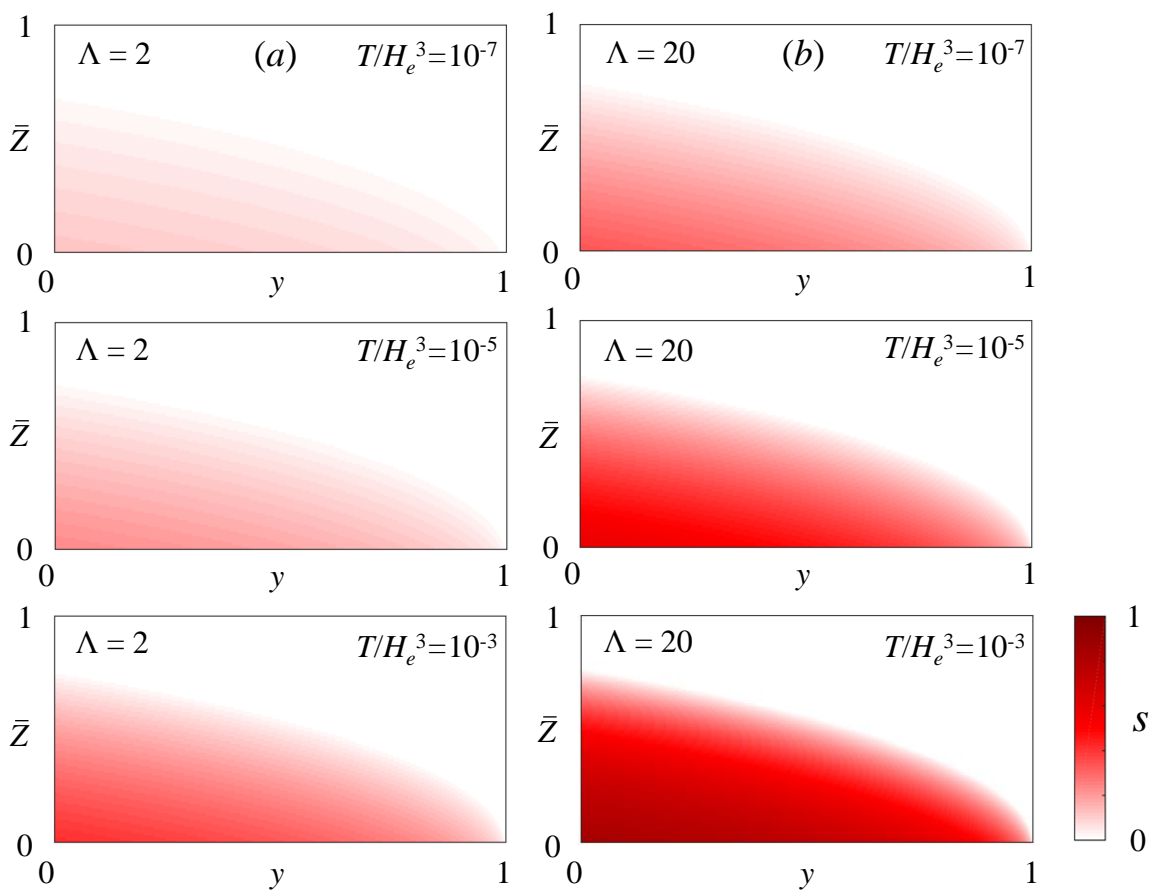

Figure 6 . The saturation field (3.19) in the early time strong capillarity regime: $(a) \Lambda=2$ and $T / H_{e}^{3}=\left\{10^{-7}, 10^{-5}, 10^{-3}\right\} ;(b) \Lambda=20$ and $T / H_{e}^{3}=\left\{10^{-7}, 10^{-5}, 10^{-3}\right\}$. A smaller $T / H_{e}^{3}$ corresponds to stronger capillary effects while a smaller $\Lambda$ corresponds to a more polydispersed pore size distribution. Both the effects of capillary forces and polydispersed pore size reduce the saturation of the injected fluid, as demonstrated here.

where ' denotes differentiation with respect to $y$. The asymptotic behaviour of $(3.15 a)$ near the front, $y=1$, is

$$
f \sim\left(\frac{2}{3}\right)^{1 / 2}(1-y)^{1 / 2}
$$

which then provides two boundary conditions $f(1-\epsilon)$ and $f^{\prime}(1-\epsilon)$ with $\epsilon \ll 1$. A shooting procedure is then employed to solve (3.15) from $y=1-\epsilon$ toward $y=0$ (here we use MATLAB's ODE45 subroutine) to obtain the solution for $f(y)$, as shown in figure $5 a$. From $(3.15 c)$ we determine the value of the constant $\xi_{f} \approx 1.48$. The location of the propagating front $X_{f}(T)$ and the vertical reach $H_{f}(T) \equiv H(0, T)$ are therefore

$$
\begin{aligned}
& X_{f}(T) \sim 1.03 T^{2 / 3}, \\
& H_{f}(T) \sim 1.37\left(H_{e} / \Lambda\right)^{1 / 2} T^{1 / 6} .
\end{aligned}
$$

We also note that the form of (3.13) and (3.14) suggests that we can define a transformation

$$
\begin{aligned}
\tilde{X} & \equiv 3^{1 / 3} X, \\
\tilde{H} & \equiv 3^{-1 / 3}\left(\Lambda / H_{e}\right) H^{2},
\end{aligned}
$$

such that $\tilde{H}(\tilde{X}, T)$ satisfies the well-known nonlinear diffusion equation for a sharpinterface gravity current in an unconfined porous medium (e.g., Huppert \& Woods 1995), see also (3.20) and (3.21) in $§ 3.3 .2$. 
Once the profile shape $H(X, T)$ is obtained, the saturation field $s[H(X, T), Z]$ can be calculated according to (3.10). Specifically, in the strong capillarity regime, defining $Z \equiv \xi_{f} 3^{1 / 6}\left(H_{e} / \Lambda\right)^{1 / 2} T^{1 / 2} \bar{Z},(3.10)$ implies that

$$
s[H(X, T), Z]= \begin{cases}1-\left[1+\xi_{f} 3^{1 / 6}\left(\frac{T}{H_{e}^{3}}\right)^{1 / 6} \Lambda^{-1 / 2}(f(y)-\bar{Z})\right]^{-\Lambda}, & 0 \leqslant \bar{Z} \leqslant f, \\ 0, & \bar{Z} \geqslant f .\end{cases}
$$

This indicates that the saturation field depends on $H_{e}, \Lambda$ and also $T$ in the early-time, strong-capillarity regime. In particular, $H_{e}$ and $T$ function together as a group $T / H_{e}^{3}$, and this is physically plausible, since a greater capillary length $H_{e}$ and a smaller time $T$ both indicate greater capillary effects. The influence of $T / H_{e}^{3}$ and $\Lambda$ on the saturation field, $s[H(X, T), Z]$, are shown in figure 6 in the early-time, strong capillarity regime, which indicates that both the effects of capillarity and pore size distribution reduce the saturation of the injected fluid.

\subsubsection{Gravity current regime: $H \gg H_{e}$}

As time progresses, the vertical extent of the current increases such that $H \gg H_{e}$ and the capillary effects become weak. For $H_{e} \ll H \ll 1$, before the confinement effects become important, (3.11) reduces to

$$
\frac{\partial H}{\partial T}-\frac{\partial}{\partial X}\left(H \frac{\partial H}{\partial X}\right)=0,
$$

which is the well-known nonlinear diffusion equation that describes the interface dynamics of a sharp-interface gravity current in an unconfined porous medium (e.g., Boussinesq 1904; Barenblatt 1952; Bear 1972; Huppert \& Woods 1995). In this limit, global mass conservation, (3.12), reduces to

$$
\int_{0}^{X_{f}(T)} H \mathrm{~d} X=T .
$$

A self-similar solution can be obtained for this system (Huppert \& Woods 1995) with $X \propto T^{2 / 3}$ and $H \propto T^{1 / 3}$, which we review here for completeness. We define a similarity variable $\eta \equiv X / T^{2 / 3}$ such that the front location is given by $X_{f}(T)=\eta_{f} T^{2 / 3}$. In terms of a normalized variable $y \equiv X / X_{f}(T)=\eta / \eta_{f}$, we may write the solution as $H(X, T)=$ $\eta_{f}^{2} T^{1 / 3} g(y)$, where $g(y)$ and $\eta_{f}$ can be found by solving

$$
\begin{array}{r}
\left(g g^{\prime}\right)^{\prime}+\frac{2}{3} y g^{\prime}-\frac{1}{3} g=0, \\
g(1)=0, \\
\eta_{f}=\left[\int_{0}^{1} g(y) \mathrm{d} y\right]^{-1 / 3} .
\end{array}
$$

The asymptotic behaviour near the front, $y=1$, is

$$
g(y) \sim \frac{2}{3}(1-y),
$$

which provides two boundary conditions $g(1-\epsilon)$ and $g^{\prime}(1-\epsilon)$ with $\epsilon \ll 1$, and a shooting procedure is used to solve (3.22) from $y=1-\epsilon$ toward $y=0$. The solution is shown in figure $5 b$, from which the constant $\eta_{f}=1.48$ is determined numerically. The location of 

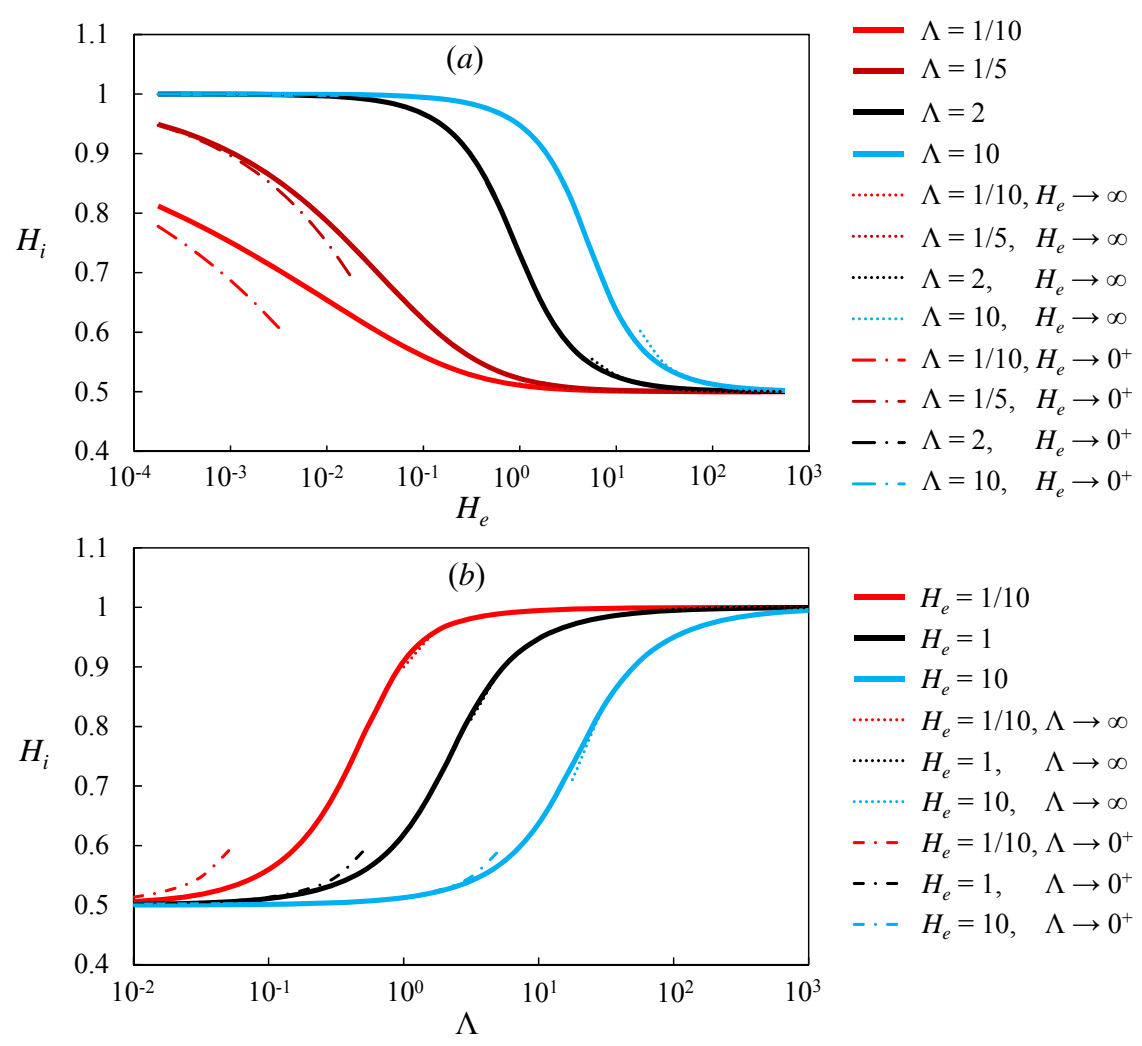

Figure 7 . Influence of $\Lambda$ and $H_{e}$ on the inlet height $H_{i}$ at the origin. The asymptotic solutions $(3.28 a)$ as $H_{e} \rightarrow 0^{+}$or $\Lambda \rightarrow \infty$, and $(3.28 b)$ as $H_{e} \rightarrow \infty$ or $\Lambda \rightarrow 0^{+}$are also shown as the dash-dotted and dotted curves, respectively.

the propagating front $X_{f}(T)$ and the vertical extent $H_{f}(T)$ is therefore given by

$$
\begin{aligned}
& X_{f}(T) \sim 1.48 T^{2 / 3}, \\
& H_{f}(T) \sim 1.30 T^{1 / 3},
\end{aligned}
$$

as found previously by (e.g., Huppert \& Woods 1995).

\subsubsection{Transition time between early time regimes}

At early times we have now identified two regimes, in which capillary forces or buoyancy dominate the dynamics of the spreading current. A simple estimate of the transition between these two regimes can be constructed from an estimate of the transition between the two height scales given by $(3.17 b)$ and $(3.24 b)$ in the capillary and gravity current regimes, respectively. The balance suggests that

$$
T_{t} \approx\left(H_{e} / \Lambda\right)^{3} .
$$

Therefore, a greater $H_{e}$, or a smaller $\Lambda$, both suggesting stronger capillary effects, would result in a greater transition time $T_{t}$. We also note that to ensure unconfined flow, we require that $H \ll 1$, which is only satisfied if $T_{t} \ll 1$. This places a constraint on the values of $H_{e}$ and $\Lambda$ for the transition to be observed in the early time period. 


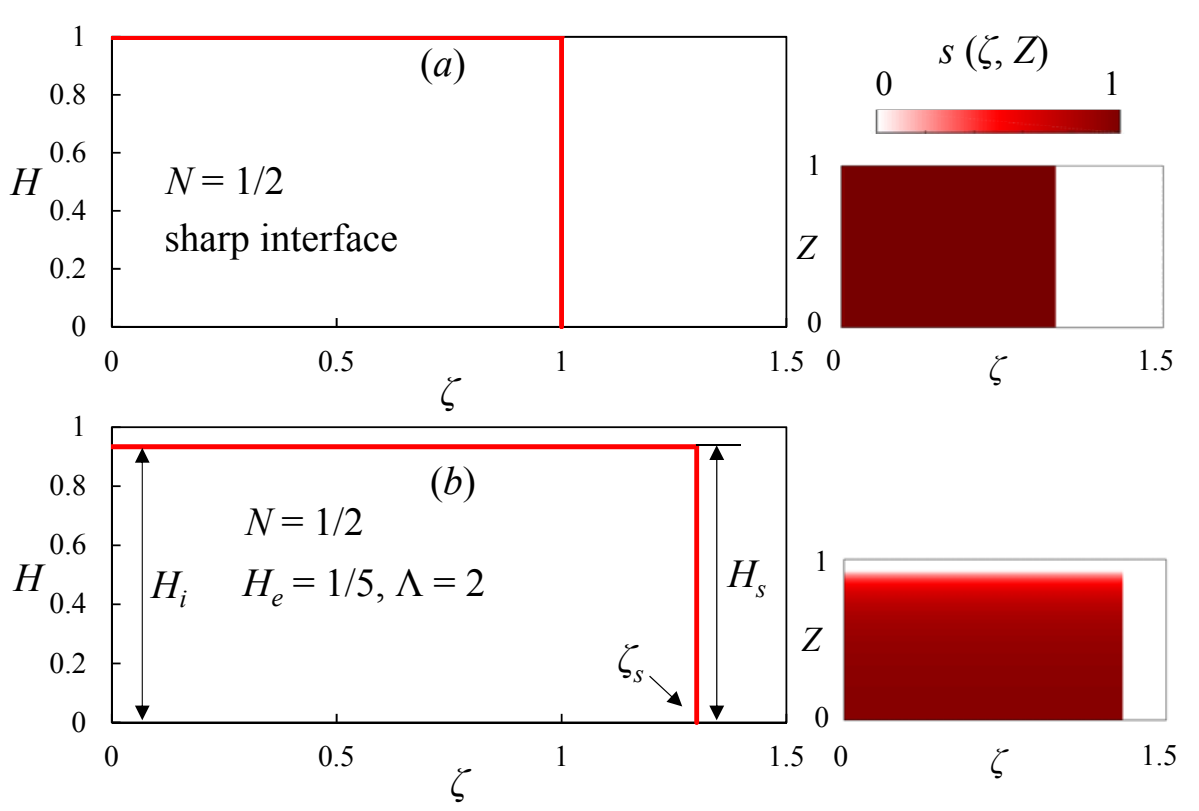

FiguRE 8. Late-time similarity solutions for $N=1 / 5$ : $(a)$ shock solution (3.30) in the sharp-interface limit, and $(b)$ modified shock solution with height $H_{s}=H_{i} \approx 0.934<1$ and front location $\zeta_{s} \approx 1.30$. The saturation field is also computed according to (3.10) and shown next to the similarity solutions.

\subsection{Late-time asymptotic solutions}

At late times $T \gg 1$, the length of the current $X \gg 1$, and the pressure gradient in the ambient fluid associated with injection can no longer be neglected. In this limit, we first examine the effects of confinement by neglecting buoyancy driven flows. In this case, (3.4) reduces to a nonlinear hyperbolic equation,

$$
\mathcal{F}_{s}(H) \frac{\partial H}{\partial T}+\frac{\partial}{\partial X}\left[\frac{N \mathcal{F}_{n}(H)}{N \mathcal{F}_{n}(H)+\mathcal{F}_{w}(H)}\right]=0 .
$$

We note again that (3.26) is analogous to the well-known Buckley-Leverett equation for partially saturating two-phase flows in a porous medium (Buckley \& Leverett 1942). Standard theory for hyperbolic conservative laws can be used to study the analytical behaviours of the equation (e.g., LeVeque 2002).

\subsubsection{The inlet thickness $H_{i}$}

We first note that the form of (3.26) suggests that $X \propto T$ for $T \gg 1$, and the inlet thickness approaches a constant $H \sim H_{i}$ at $X=0$. In this case, boundary condition (3.9) reduces to

$$
\left(1-H_{i}\right)+\frac{H_{e}}{1-2 \Lambda}\left[1-\left(1+\frac{H_{i}}{H_{e}}\right)^{1-2 \Lambda}\right]=0
$$

which indicates that the inlet thickness $H_{i}$ depends on the capillary height $H_{e}$ and the pore-size distribution parameter $\Lambda$ and is independent of the modified viscosity ratio $N$. The influence of $H_{e}$ and $\Lambda$ on $H_{i}$ is calculated numerically from (3.27) and is shown in figure 7. Explicit expressions of $H_{i}$ are also available, for a given $\Lambda$, in the asymptotic 

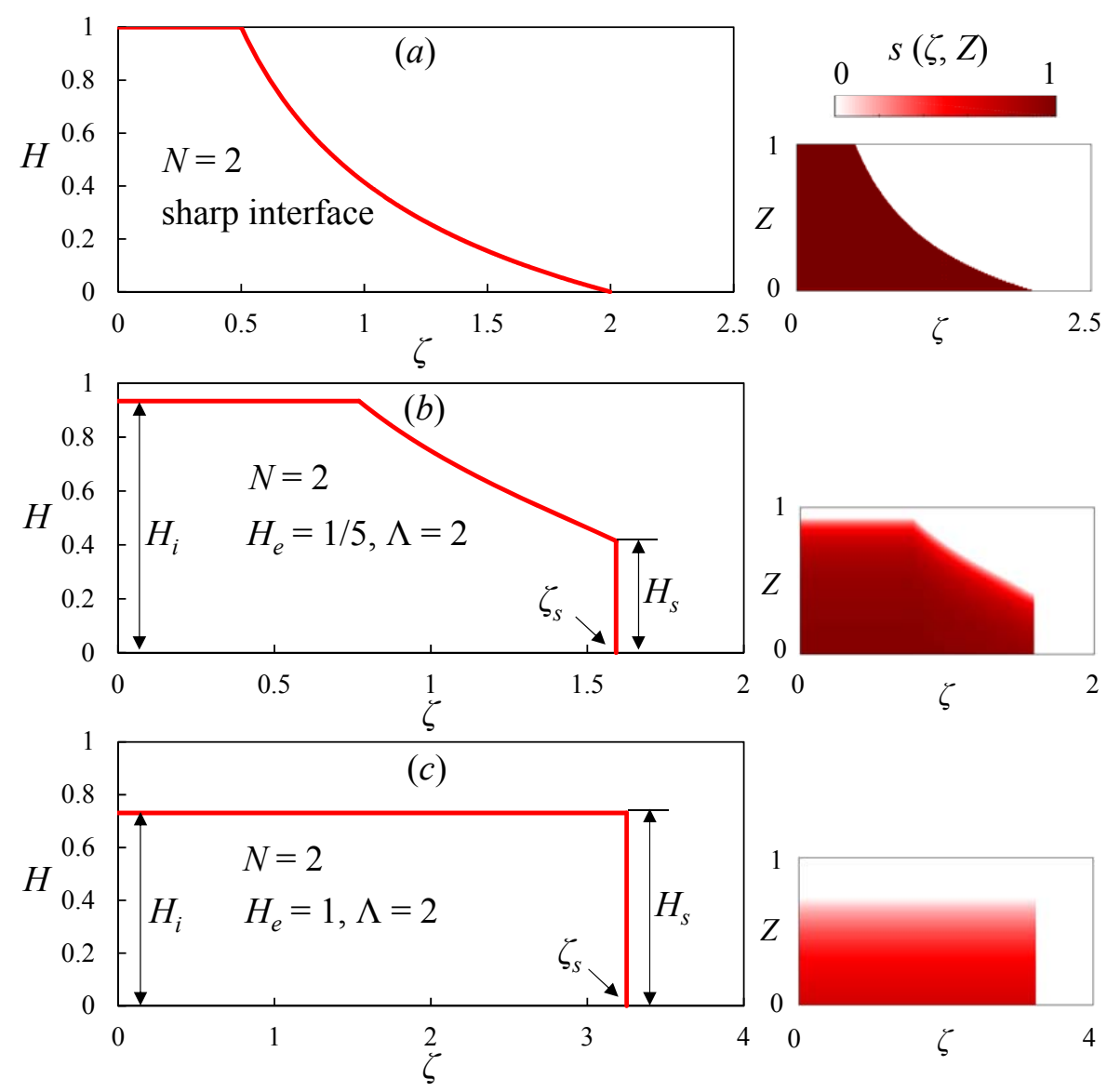

Figure 9. Late-time similarity solutions for $N=2, \Lambda=2$ and $H_{e}=\{1 / 5,1\}$ : (a) rarefaction solution (3.31) in the sharp-interface limit, $(b)$ compound wave solution with $H_{i} \approx 0.934$, $H_{s} \approx 0.414$ and $\zeta_{s} \approx 1.59$, and $(c)$ modified shock solution with $H_{s}=H_{i} \approx 0.731<1$ and front location $\zeta_{s} \approx 3.24$. The saturation field is also computed according to (3.10) and shown next to the similarity solutions.

limits of $H_{e} \rightarrow 0^{+}$(weak capillarity) and $H_{e} \rightarrow \infty$ (strong capillarity), or, for a given $H_{e}$, in the asymptotic limit of $\Lambda \rightarrow 0^{+}$(polydispersed pore size distribution) and $\Lambda \rightarrow \infty$ (monodispersed pore size distribution). These expressions,

$$
\begin{aligned}
H_{i} & \sim 1+\frac{H_{e}}{1-2 \Lambda}-\frac{H_{e}^{2 \Lambda}}{(1-2 \Lambda)\left(H_{e}+1\right)^{2 \Lambda-1}} \sim 1, \text { as } H_{e} \rightarrow 0^{+} \text {or } \Lambda \rightarrow \infty, \\
H_{i} & \sim\left(\frac{\Lambda}{H_{e}}\right)^{-1}\left[1-\left(1-\frac{\Lambda}{H_{e}}\right)^{1 / 2}\right] \sim \frac{1}{2}, \quad \text { as } H_{e} \rightarrow \infty \text { or } \Lambda \rightarrow 0^{+},
\end{aligned}
$$

are plotted as the dotted and dot-dashed curves, respectively, in figure 7 . The asymptotic result $(3.28 a)$ in the weak capillarity or monodisperse pore size limit indicates that the interface contacts the top boundary and recovers the sharp interface limit (Pegler et al. 2014; Zheng et al. 2015a). In comparison, in the strong capillarity, or broad pore-size distribution limit, (3.28b) indicates that the interface does not contact the top boundary, which provides a major difference from the sharp interface limit and can be of importance 

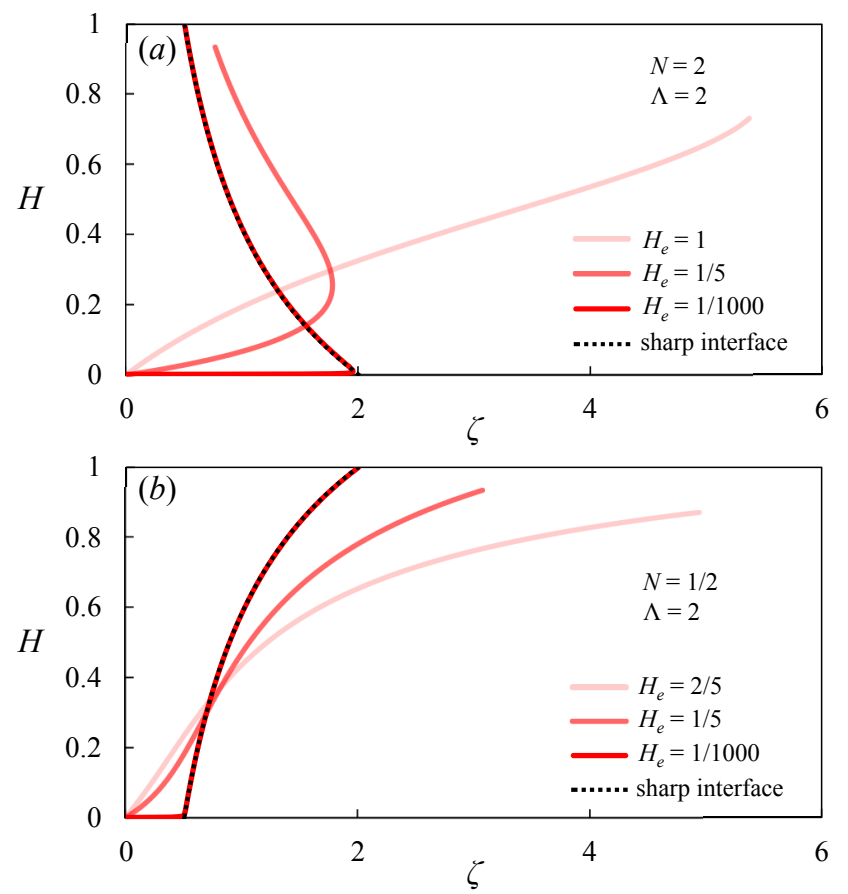

Figure 10. Representative flux functions $\zeta=F(H)$, as defined in (3.33), for different $N, \Lambda$ and $H_{e}$. The corresponding flux functions in the sharp interface limit, based on (3.29), are plotted as the dashed curve. (a) With $N=2$ and $\Lambda=2, F(H)$ is non-monotonic for $H_{e}=\{1 / 1000,1 / 5\}$ while increases monotonically for $H_{e}=1$. (b) With $N=1 / 2$ and $\Lambda=2, F(H)$ increases monotonically for all $H_{e}$. The flux functions with the same viscosity ratio $N$ in the sharp interface limit are also plotted in both $(a)$ and $(b)$.

for practical applications such as geological $\mathrm{CO}_{2}$ sequestration, as we discussed in detail in $\S 6$.

\subsubsection{Sharp interface limit: $H_{e} \rightarrow 0^{+}$or $\Lambda \rightarrow \infty$}

When $H_{e} \rightarrow 0^{+}$or $\Lambda \rightarrow \infty$, the capillary effects are weak and the pore size is effectively monodisperse for the confined two-phase flow. In this asymptotic limit, (3.26) reduces to

$$
\frac{\partial H}{\partial T}+\frac{\partial}{\partial X}\left[\frac{N H}{(N-1) H+1}\right]=0,
$$

which includes only one parameter $N \equiv M k_{r n 0}$, which is the modified viscosity ratio. Equation (3.29) recovers the sharp-interface model when the capillary effects are neglected recovering, for example, equation (3.13) in Pegler et al. (2014) or equation (3.6) in Zheng et al. (2015a). The only difference is that (3.29) incorporates the endpoint permeability through the modified viscosity ratio $N$, rather than the viscosity ratio $M \equiv \mu_{w} / \mu_{n}$ in (3.13) in Pegler et al. (2014) and (3.6) in Zheng et al. (2015a).

The scalar equation (3.29) has a convex flux function, as discussed in Zheng et al. (2015a). Thus, the theory of hyperbolic conservation laws indicates that the initial condition will: (i) evolve into a shock solution when $N<1$, (ii) retain the inital shape when $N=1$, or (iii) evolve into a rarefaction solution when $N>1$. In particular, in the case of (i) and (ii), a self-similar solution can be obtained by further considering the effects 

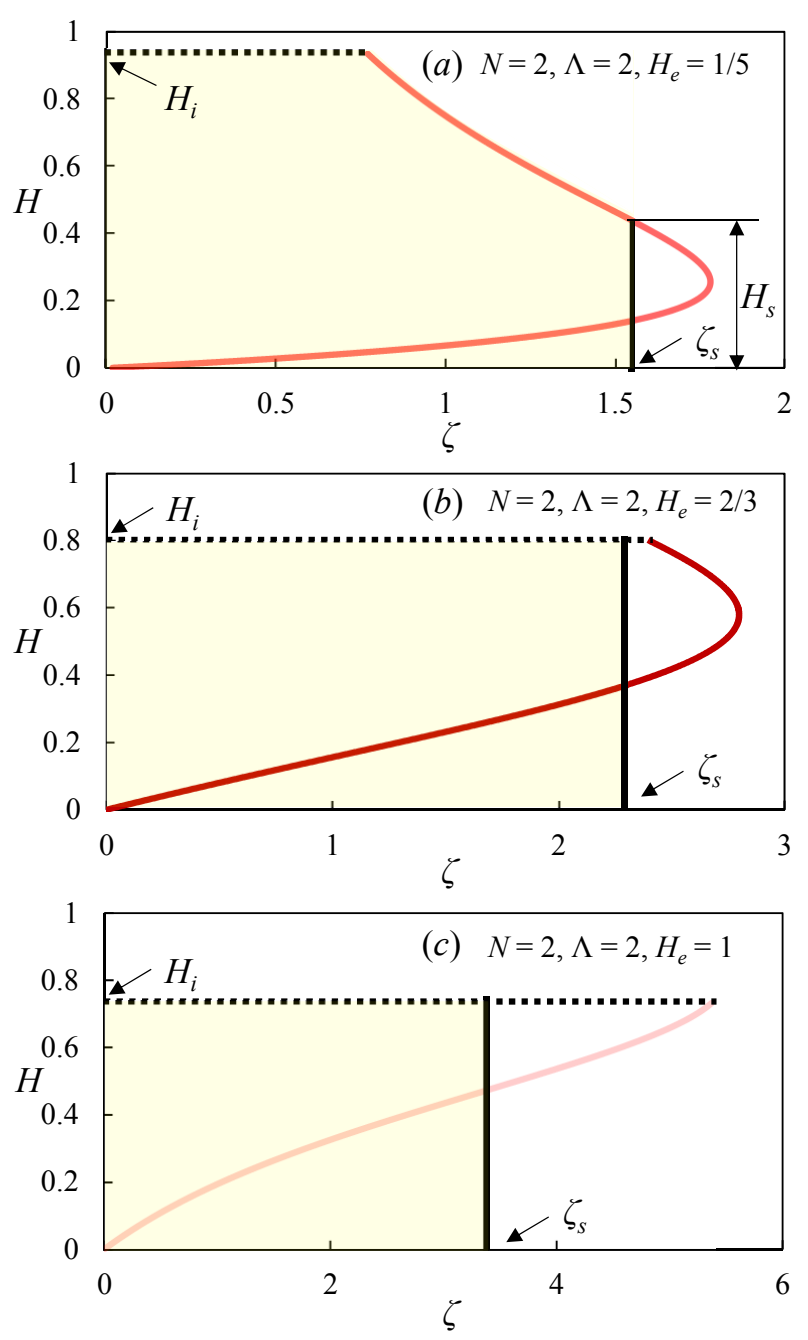

Figure 11. The location of the shock front is determined such that the amount of injected fluid in the shaded area satisfies the global mass constraint (3.34). Three scenarios are demonstrated here: $(a)$ a compound wave solution for a non-monotonic flux function $F(H),(b)$ a modified shock solution from a non-monotonic $F(H)$, and $(c)$ a modified shock solution from a monotonically increasing $F(H)$.

of buoyancy. More detailed discussions can be found in Pegler et al. (2014) and Zheng et al. $(2015 a)$.

For completeness, we review the explicit expressions for the shock and rarefaction solutions, depending on the value of $N$. The shock solution, in particular, exists when $N<1$, and is given by

$$
H(X, T)= \begin{cases}1, & X / T \leqslant 1 \\ 0, & X / T>1\end{cases}
$$

In addition, the speed of the propagating fronts attaching the bottom boundary, denoted by $X_{f}(T)$, and the top boundary, denoted by $X_{f 2}(T)$, is given by

$$
X_{f}(T)=X_{f 2}(T)=T \text {. }
$$



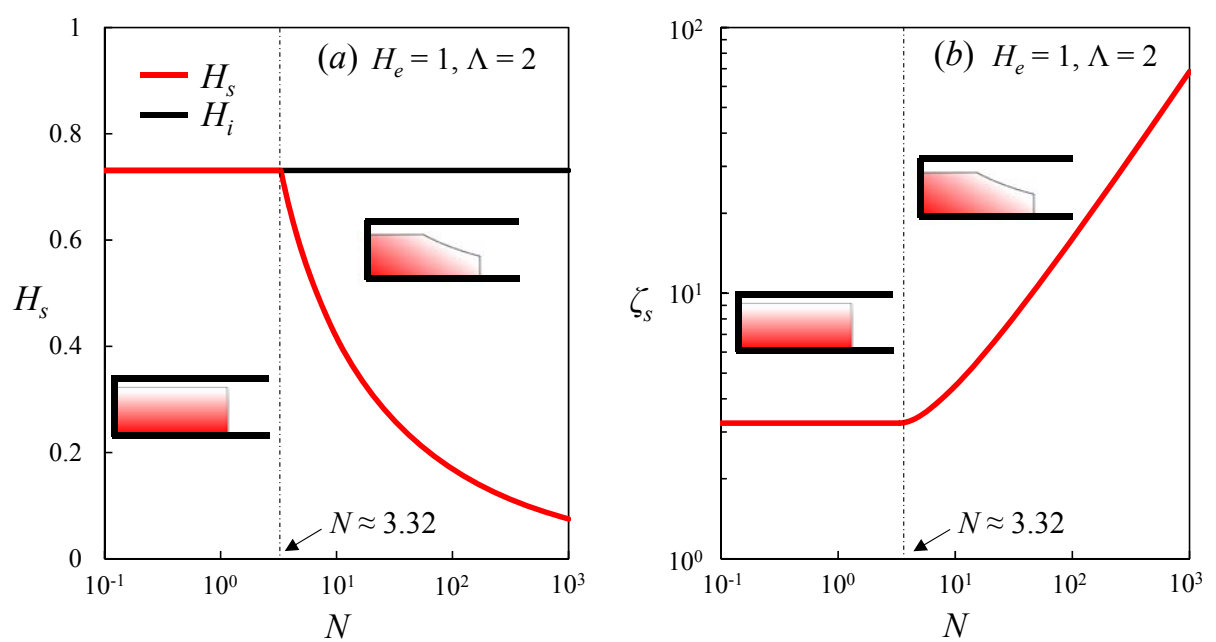

Figure 12. Influence of $N$ on the height and location of the shock front, $H_{s}$ and $\zeta_{s}$, respectively. We set $H_{e}=1$ and $\Lambda=2$ in this example. Two regimes are identified for either a compound wave or modified shock solution, separated by a critical viscosity ratio $N \approx 3.32$ as the regime boundary.

When $N>1$, in comparison, the rarefaction solution is used to describe the evolution of the interface shape $H(X, T)$, which can be written as

$$
H(X, T)= \begin{cases}1, & X / T \leqslant 1 / N ; \\ (\sqrt{N /(X / T)}-1) /(N-1), & 1 / N<X / T \leqslant N ; \\ 0, & X / T>N .\end{cases}
$$

The location of the propagating fronts along the bottom and top boundaries may also be computed as

$$
X_{f}(T)=N T, \text { and } X_{f 2}(T)=T / N .
$$

The rarefaction solution for $N=2$ and the shock solution for $N=1 / 2$ are shown in figure $8 a$ and figure $9 a$, respectively.

\subsubsection{Similarity solutions in the advective limit}

In the advective limit, in which buoyancy-driven flow is negligible, we find a series of self-similar solutions which depend on the effective viscosity ratio $N$, the capillary height $H_{e}$ and the pore-size distribution $\Lambda$. We now investigate the original hyperbolic evolution equation, (3.26), and explore the influence of control parameters $N, H_{e}$ and $\Lambda$. We first define a similarity variable as $\zeta \equiv X / T$ and hence $H(X, T)=H(\zeta)$. Then, (3.26) becomes

$$
\zeta=F(H) \equiv \frac{1}{\mathcal{F}_{s}(H)} \frac{\partial}{\partial H}\left[\frac{N \mathcal{F}_{n}(H)}{N \mathcal{F}_{n}(H)+\mathcal{F}_{w}(H)}\right],
$$

subject to global mass conservation which, according to $(2.15)$, becomes

$$
\int_{0}^{\zeta_{s}}\left(H+\frac{H_{e}}{1-\Lambda}\left[1-\left(1+\frac{H}{H_{e}}\right)^{1-\Lambda}\right]\right) \mathrm{d} \zeta=1
$$

where $\zeta_{s} \equiv X_{f} / T$ is the location of the shock front.

Depending on the values of $N, H_{e}$ and $\Lambda$, two types of similarity solutions $H(\zeta)$ are 

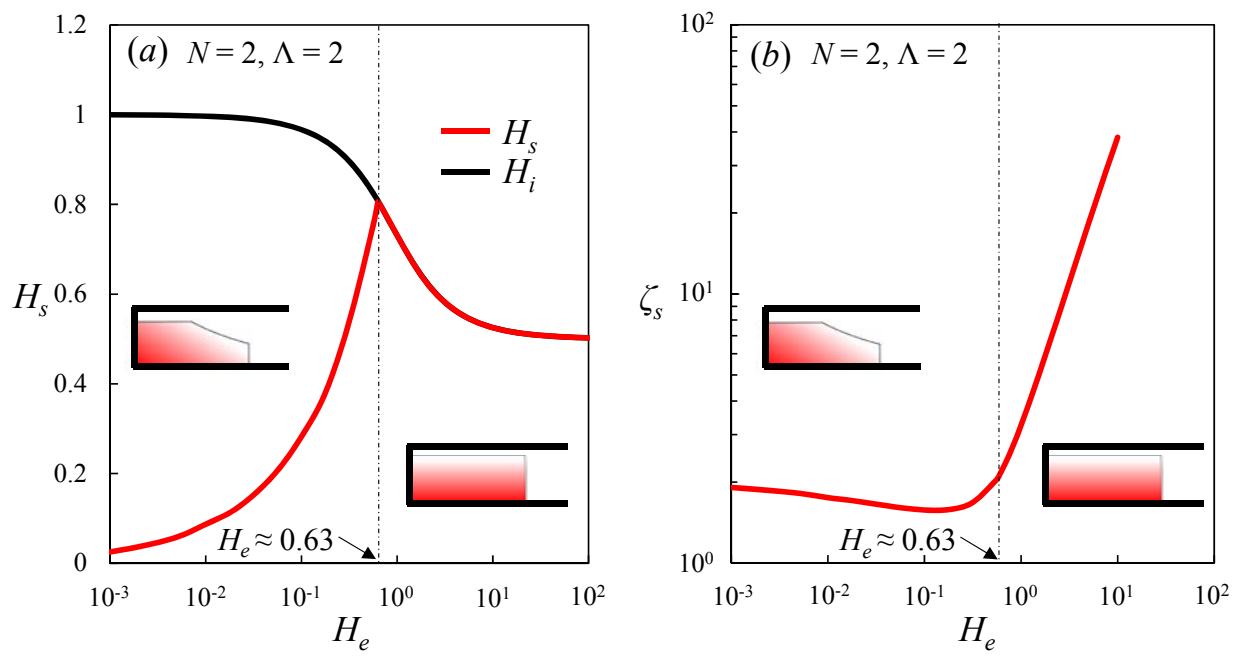

Figure 13. Influence of $H_{e}$ on the height $H_{s}$ and location $\zeta_{s}$ of the shock front. We set $N=2$ and $\Lambda=2$ in this example, and identify two regimes that correspond to either a compound wave a modified shock solution. A critical capillary length $H_{e} \approx 0.63$, which sets the regime boundary, is calculated for this example.

available; (i) a compound wave solution, which includes a stretching region and a shock front (see figure $9 b$ ) and (ii) a modified shock solution with an inlet thickness $H_{i}<1$ (see figure $8 b$ and figure $9 c$ ). Here the word "modified" is simply used in contrast to the shock solution, (3.30), with $H_{i}=1$ in the sharp-interface limit. In addition, the saturation field now becomes $s[H(X, T), Z]=s(\zeta, Z)$. With the interface shape $H(\zeta)$ available, $s(\zeta, Z)$ is then computed according to (3.10) and is also shown next to the similarity solutions in figures 8 and 9.

We note that the similarity solution $H(\zeta)$ is related to the form of the flux function $F(H)$ defined in (3.33). Representative calculations of the flux function $F(H)$ are shown in figure 10 for particular sets of $N, \Lambda$ and $H_{e} . F(H)$ exhibits two different trends, depending on $N, \Lambda$ and $H_{e}$; (i) $F(H)$ increases monotonically with $H$, and (ii) $F(H)$ is non-monotonic and reaches a maximum between $H=0$ and $H=H_{i}$. The flux functions in the sharp-interface limit for the same viscosity ratio $N$ are also shown as the dashed curves in figure 10, which is approached as $H_{e} \rightarrow 0^{+}$with major difference near $H=0$.

The construction of these similarity solutions is demonstrated in figure $11 a$ for a compound wave solution and in figure $11 b, c$ for a modified shock solution. The location of the shock fronts $\left(\zeta_{s}\right)$ in both cases is determined such that the global mass constraint (3.34) is satisfied. We note that the "equal-area" rule (e.g., Chapter 11, LeVeque 2002), as employed in previous studies (e.g., Taghavi et al. 2009; Zheng et al. 2015b), does not apply in the present problem since the saturation of the injected fluid varies along the vertical direction because of capillary effects. In addition, the inlet thickness $H_{i}$ is calculated according to (3.27), or (3.28) in the asymptotic limits of $H_{e} \rightarrow 0^{+}$or $\Lambda \rightarrow \infty$.

The influence of the dimensionless control parameters $N, \Lambda$ and $H_{e}$ on the location $\left(\zeta_{s}\right)$ and height $\left(H_{s}\right)$ of the shock front are demonstrated in figures 12 and 13. In particular, two regimes can be identified, which correspond to either a compound wave or a modified shock solution. For example, with $H_{e}=1$ and $\Lambda=2$, the critical viscosity ratio $N \approx 3.32$ distinguishes the two types of solutions, as shown in figure 12 . In addition, with $N=2$ 


\begin{tabular}{cccccc}
\hline Items & Case 1 & Case 2 & Case 3 & Case 4 & Case 5 \\
Parameters: & & & & & \\
$N$ & 2 & 2 & 2 & $1 / 2$ & $1 / 2$ \\
$\Lambda$ & 2 & 2 & 2 & 2 & 2 \\
$H_{e}$ & $10^{-3}$ & $1 / 5$ & 1 & $10^{-3}$ & $1 / 5$
\end{tabular}

Early-time unconfined flows:

when $T \ll\left(H_{e} / \Lambda\right)^{3}$,

$\begin{array}{lccccc}\text { Similarity } & \mathrm{C} & \mathrm{C} & \mathrm{C} & \mathrm{C} & \mathrm{C} \\ X_{f} & \sim 1.03 T^{\frac{2}{3}} & \sim 1.03 T^{\frac{2}{3}} & \sim 1.03 T^{\frac{2}{3}} & \sim 1.03 T^{\frac{2}{3}} & \sim 1.03 T^{\frac{2}{3}} \\ H_{f} & \sim 0.0306 T^{\frac{1}{6}} & \sim 0.433 T^{\frac{1}{6}} & \sim 0.969 T^{\frac{1}{6}} & \sim 0.0306 T^{\frac{1}{6}} & \sim 0.433 T^{\frac{1}{6}}\end{array}$

when $\left(H_{e} / \Lambda\right)^{3} \ll T \ll 1$,

$\begin{array}{lcccc}\text { Similarity } & \mathrm{B} & - & - & \mathrm{B} \\ X_{f} & \sim 1.48 T^{\frac{2}{3}} & - & - & \sim 1.48 T^{\frac{2}{3}} \\ H_{f} & \sim 1.30 T^{\frac{1}{3}} & - & - & \sim 1.30 T^{\frac{1}{3}}\end{array}$

Late-time confined flows:

when $T \gg 1$,

$\begin{array}{llcccc}\text { Similarity } & \text { CW } & \text { CW } & \text { MS } & \text { MS } & \text { MS } \\ X_{f} & \sim 1.91 T & \sim 1.59 T & \sim 3.24 T & \sim 1.00 T & \sim 1.30 T \\ H_{i} & \sim 1.00 & \sim 0.934 & \sim 0.731 & \sim 1.00 & \sim 0.934\end{array}$

TABLE 2. Summary of control parameters and asymptotic behaviours for solutions to (3.4) in $\S 4$. Here $X_{f}$ is the front location, $H_{f}$ is the vertical reach and $H_{i}$ is the time-indepdent inlet thickness at late times. For early-time unconfined flows, "C" represents a capillarity similarity solution (§3.3.1) and "B" represents a buoyancy similarity solution (§3.3.2). For late-time confined flows, "CW" represents the a compound wave solution $(\S 3.4)$ and "MS" represents a modified shock solution $(\S 3.4)$.

and $\Lambda=2$, a critical capillary length $H_{e} \approx 0.63$ is identified as the regime boundary, as shown in figure 13 .

We note that when $N>1$, the compound wave solution degenerates into the rarefaction solution (3.31) in the sharp interface limit for $H_{e} \rightarrow 0^{+}$(weak capillarity) or $\Lambda \rightarrow \infty$ (weak pore heterogeneity). In this case, the height of the shock front $H_{s} \rightarrow 0^{+}$, and the stretching region extends to the bottom boundary $(Z=0)$. In comparison, when $N<1$, the height of the modified shock $H_{s}=H_{i} \rightarrow 1^{-}$and the solution degenerates into the shock solution (3.30) for $H_{e} \rightarrow 0^{+}$or $\Lambda \rightarrow \infty$. 

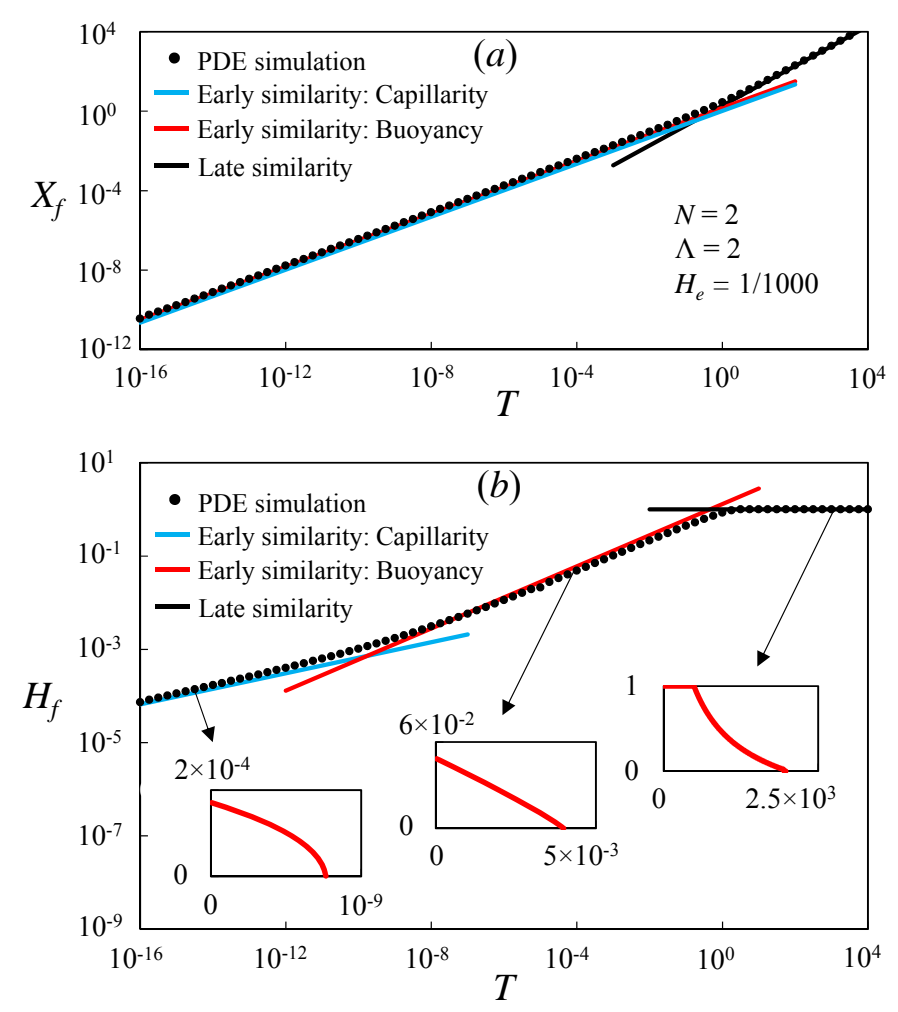

FiguRE 14. Evolution of the front location $X_{f}(T)$ in $(a)$ and vertical reach $H_{f}(T)$ in $(b)$ for $N=2, \Lambda=2$ and $H_{e}=1 / 1000$. Numerical solutions are shown as dots, while the early-time and late-time self-similar solutions are shown as straight lines. The insets in $(b)$ are the profiles at different representative times $T=\left\{10^{-14}, 10^{-4}, 10^{3}\right\}$ from the numerical solutions.

\section{Full numerical solutions}

In order to confirm the presence of the various self-similar solutions and to explain in more details the transition between the dominant physical behaviours, we numerically solve (3.4) subject to initial condition (3.7) and boundary conditions (3.8) and (3.9). We then compare the numerical results with the theoretical predictions of various similarity solutions in the early and late time periods, respectively. We also show the time transition between the different asymptotic regimes we have identified. The dimensionless control parameters we have chosen for the case studies and the corresponding asymptotic solutions and front propagation laws in each case are summarized in table 2.

A finite difference scheme, developed by Kurganov \& Tadmor (2000), was employed to solve the advective-diffusive equation, (3.4), which has been tested in previous studies of sharp-interface models of immiscible fluid displacement in porous media and horizontal channels (e.g., Zheng et al. 2015a,b; Guo et al. 2016b). For numerical convenience, we set the farfield thickness $h(x \rightarrow \infty)=\mathcal{O}\left(10^{-15}\right)$, and solve (3.4) with different domain lengths for numerical simulations spanning a wide range of time (and length) scales. Convergence tests were performed to verify that the results are independent of further mesh refreshment. 

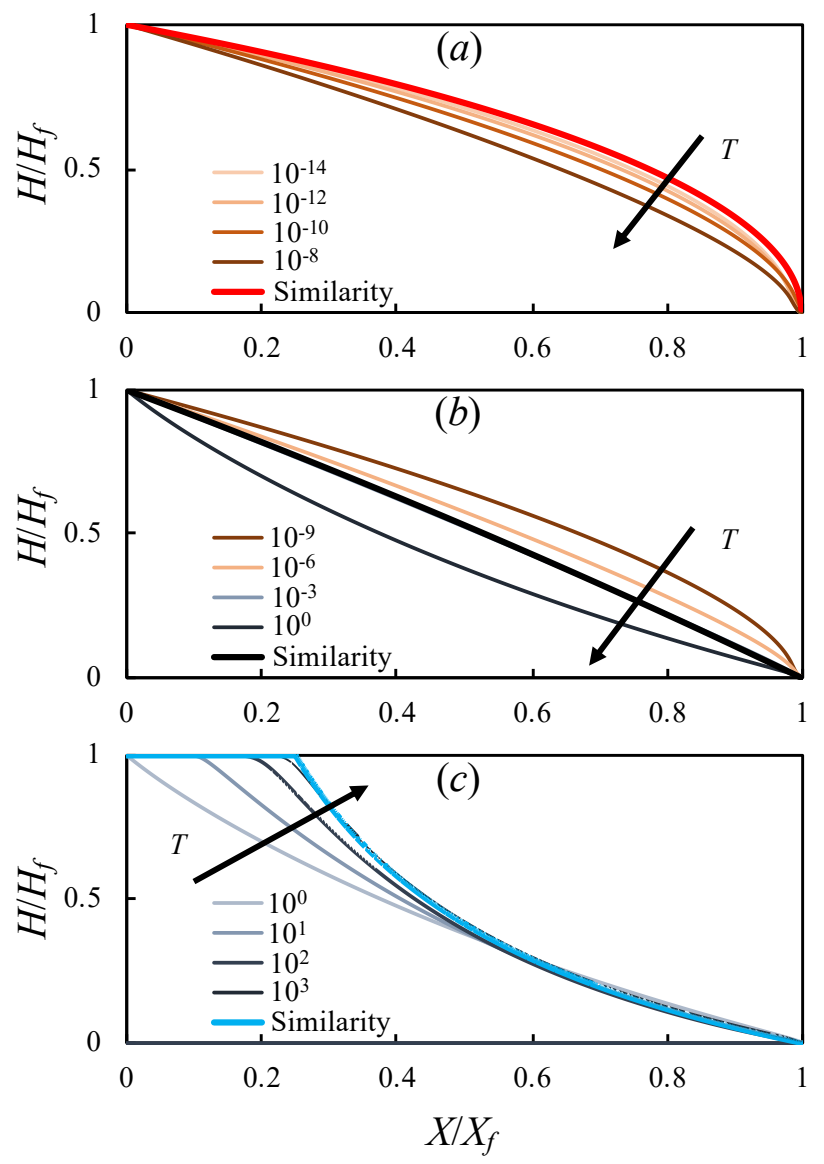

Figure 15. Evolution for the rescaled shapes with $N=2, \Lambda=2$ and $H_{e}=1 / 1000$. The PDE numerical simulation departs from the capillarity similarity solution of (3.15) in the early-time period in $(a)$, approaches the buoyancy similarity solution (3.22) at intermediate times in $(b)$, before eventually approaches the confined similarity solution in the late-time period in $(c)$.

\subsection{Time transition between early- and late-time self-similar behaviours}

In the sharp interface limit, viscosity ratios $N>1$ correspond to a rarefaction solution in the late time period. To investigate the capillary effects, we set $N=2, \Lambda=2$ and performed numerical solutions for $H_{e}=\{1 / 1000,1 / 5,1\}$. The evolution of the front location $X_{f}(T)$, vertical extent $H_{f}(T)$ and the profile shapes $H(X, T)$ are shown in figures $14-17$. We have also investigated the time transition for $N=1 / 2$, and the results and discussions can be found in Appendix B.

At early times, the capillarity similarity solution appears in all cases, as evidenced from both the numerical results for the front location (figures 14, 16, 17) and interface shape (figures $15 a, 16 c, 17 c$ ). As time progresses, the numerical solution approaches the buoyancy similarity solution at intermediate times for the case with $H_{e}=1 / 1000$ (figures 14, 15b). In comparison, for $H_{e}=\{1 / 5,1\}$, the buoyancy similarity solution does not appear in the numerical solutions (figures 16, 17). At late times, the numerical solutions approach three different late-time similarity solutions: (i) For $H_{e}=1 / 1000$, the rarefaction solution provides a good approximate (figures 14, 15c), (ii) for $H_{e}=1 / 5$, the 

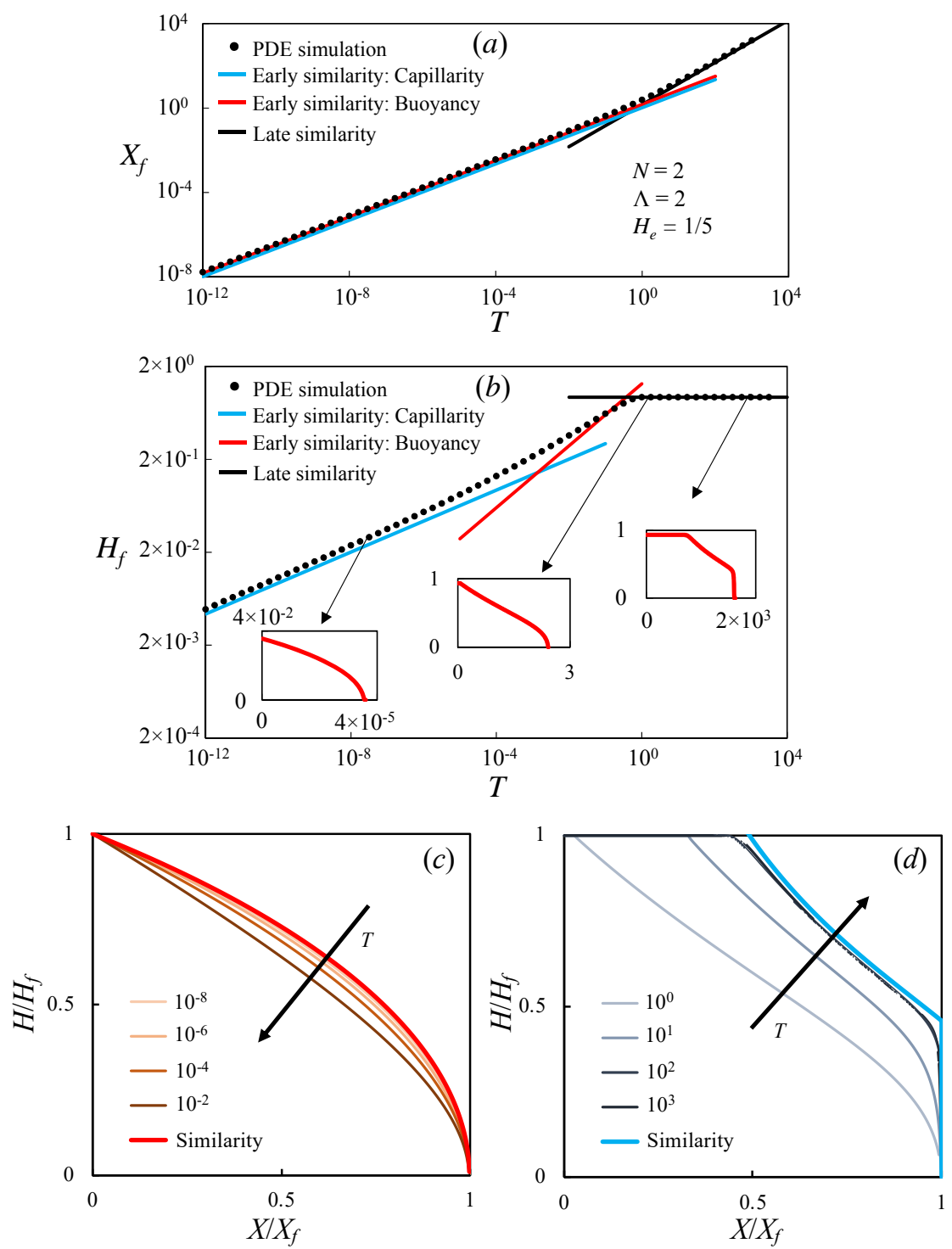

Figure 16. Evolution for the front location $X_{f}(T)$ in $(a)$, vertical reach $H_{f}(T)$ in $(b)$ and rescaled profile shapes in $(c, d)$ for $N=2, \Lambda=2$ and $H_{e}=1 / 5$. In $(a, b)$, the numerical solutions are shown as dots, while the early-time and late-time self-similar solutions are shown as straight lines. The insets in $(b)$ are the profiles at different representative times $T=\left\{10^{-7}, 10^{0}, 10^{3}\right\}$ from numerical solutions. In $(c, d)$, the numerical solutions depart from the capillarity similarity solution of (3.15) in the early-time period in $(a)$, while they approach the confined similarity solution (compound wave) in the late-time period in $(b)$.

numerical solutions approach a compound wave solution (figure 16a,b,d), and (iii) for $H_{e}=1$, the numerical solutions approach a modified shock solution (figure $17 a, b, d$ ). 

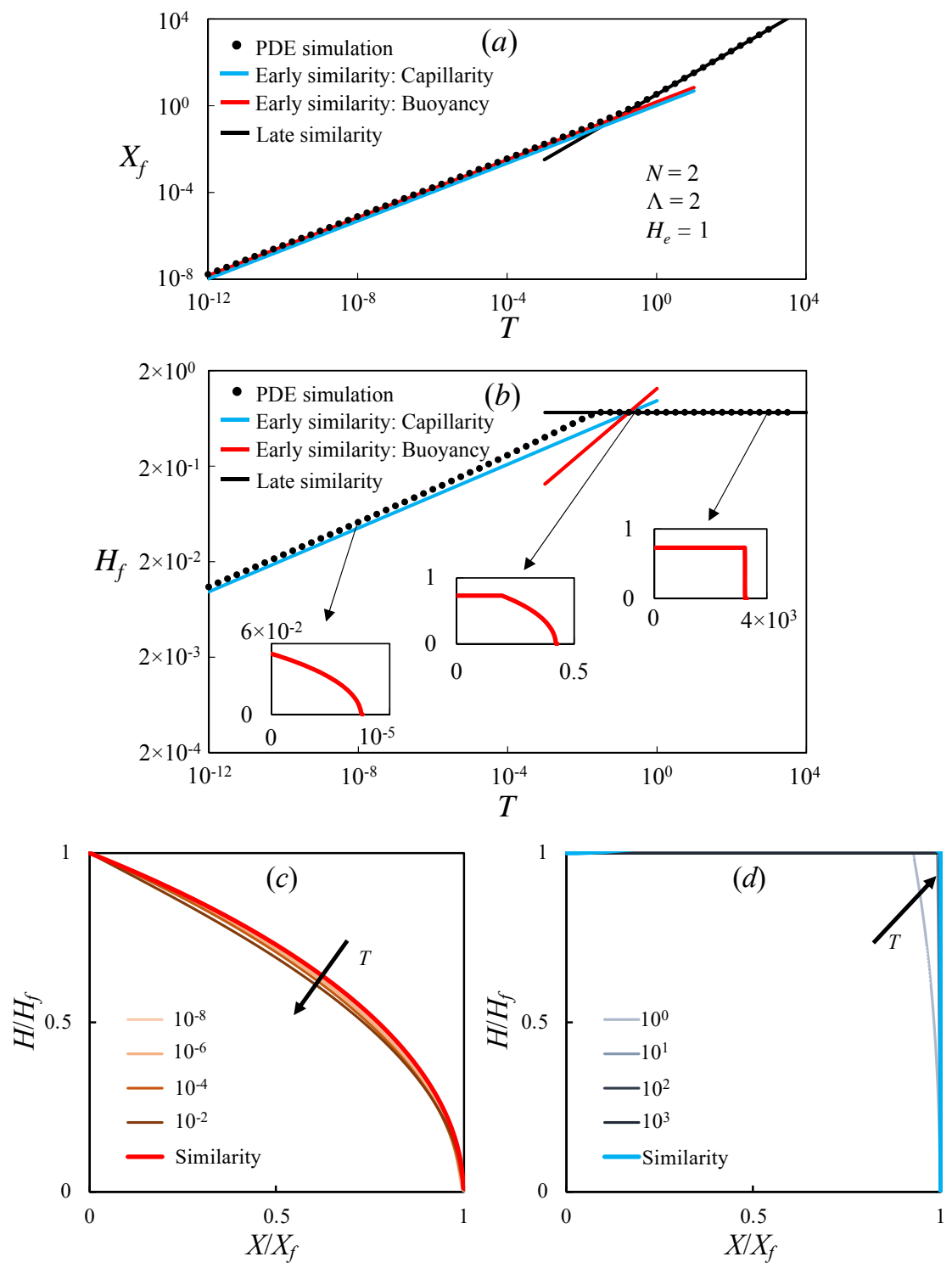

FiguRE 17. Evolution for the front location $X_{f}(T)$ in $(a)$, vertical reach $H_{f}(T)$ in $(b)$ and profile shapes in $(c, d)$ for $N=2, \Lambda=2$ and $H_{e}=1$. In $(a, b)$, the numerical solutions are shown as dots, while the early-time and late-time self-similar solutions are shown as straight lines. The insets in $(b)$ are the profiles at different representative times $T=\left\{10^{-8}, 10^{-1}, 10^{3}\right\}$ from numerical solutions. In $(c, d)$, the numerical solutions depart from the capillarity similarity solution of (3.15) in the early-time period in $(c)$, while they approach the confined similarity solution (modified shock) in the late-time period in $(d)$.

\section{Schematic regime diagram and discussions}

\subsection{Schematic regime diagram}

A schematic regime diagram is provided in figure 18, which summarizes the evolution of the interface shape for two-phase fluid flows driven by injection into a confined porous layer. We have identified six possible similarity solutions: a capillarity solution (C) and 


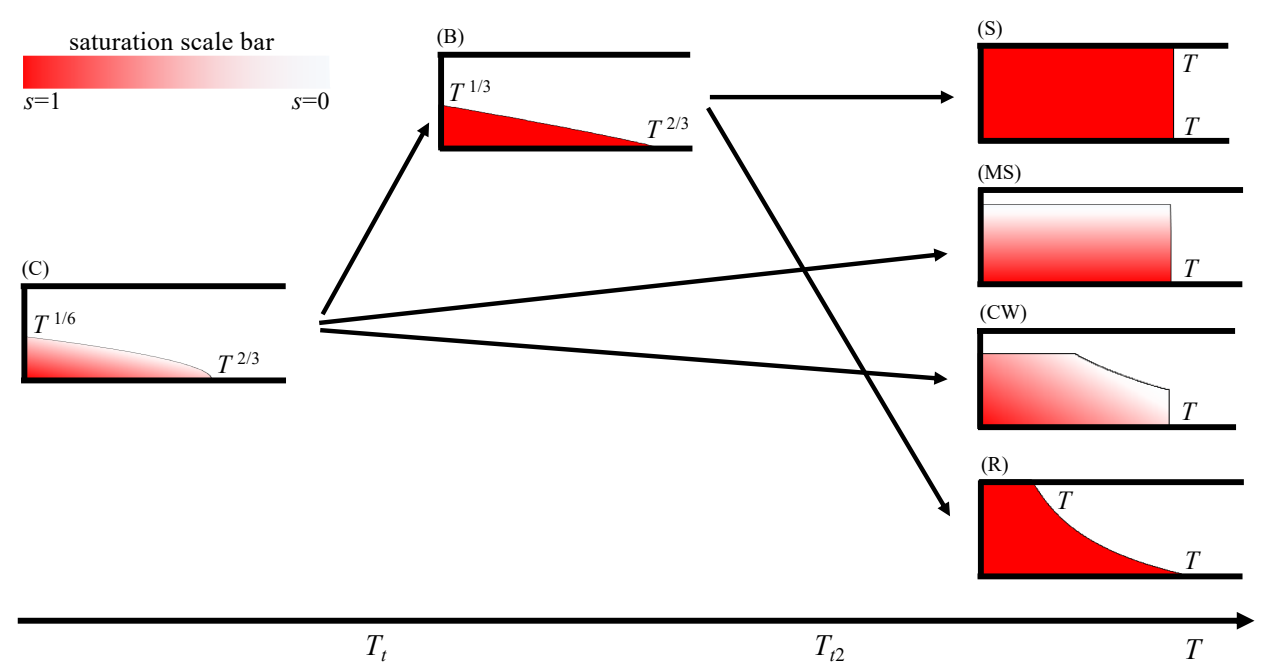

FIGURE 18. Schematic regime diagram summarizing the possible asymptotic behaviours during fluid injection into a confined porous layer. Six possible similarity solutions are identified: a capillarity solution (C) and buoyancy solution (B) for the early-time unconfined flows, and a shock solution (S), a modified shock solution (MS), a compound wave solution (CW) and a rarefaction solution $(\mathrm{R})$ for the late-time confined flows. The early-time transition time $T_{t}$ is given by (3.25), while the late-time transition time $T_{t 2}=T_{t 2}\left(N, H_{e}, \Lambda\right)$.

buoyancy solution (B) for the early-time unconfined flows, and a shock solution (S), a modified shock solution (MS), a compound wave solution (CW), and a rarefaction solution $(\mathrm{R})$ for the late-time confined flows. In the sharp-interface limit, the interface envolves from the buoyancy solution (B) to either a rarefaction solution (R) or a shock solution (S).

With capillary effects, in comparison, the flow partially saturates the porous medium and starts from an early-time capillarity solution (C) before eventually developing into either a modified shock solution (MS) or a compound wave solution (CW). We also note that, when the capillary effects are weak, the buoyancy solution (B) can appear as a good approximate to describe the flow behavour at intermediate times. In addition, the modified shock (MS) and compound wave (CW) solutions at late times reduce to the shock (S) and the rarefaction (R) solutions in the asymptotic limit of zero capillarity $\left(H_{e} \rightarrow 0^{+}\right)$. The specific pathways taken in the regime diagram (figure 18) are based on the values of the three dimensionless parameters $N, H_{e}$ and $\Lambda$, as we describe in more detail in $\S 5.2$.

\subsection{Influence of control parameters $N, H_{e}$ and $\Lambda$}

The influence of dimensionless parameters $N, H_{e}$ and $\Lambda$ on the behaviour of similarity solutions in the schematic regime diagram (figure 18) is summarised in table 3.

In particular, in the early-time period, for the capillarity similarity solution $(\mathrm{C})$, the universal shape $f(y)$ and the location of the propagating front $X_{f}(T)$ are both independent of $N, H_{e}$ and $\Lambda$, as calculated from (3.15) and (3.17a). However, the vertical front $H_{f}$, given by $(3.17 b)$, scales with $\left(H_{e} / \Lambda\right)^{1 / 2}$. For the buoyancy similarity solution (B), the universal shape $g(y)$, the front locations $X_{f}$ and $H_{f}$ are all independent of the control parameters $N, H_{e}$ and $\Lambda$.

In the late-time period, in comparison, the flow is confined, and the similarity solutions in $\S 3.4$ can be influenced by $N, H_{e}$ and $\Lambda$. In the limit of negligible capillary effects, the 
Similarity solutions

Early-time unconfined flows:

Capillarity (C)

Buoyancy (B)

Late-time confined flows:

Shock (S)

Modified shock (MS)

Compound wave (CW)

Rarefaction ( $\mathrm{R})$
Items

$N \quad H_{e}$

$\Lambda$

$\begin{array}{llll}\text { Universal shape } f(y) & \boldsymbol{x} & \boldsymbol{x} & \boldsymbol{x} \\ \text { Front location } X_{f}(T) & \boldsymbol{x} & \boldsymbol{x} & \boldsymbol{x} \\ \text { Vertical reach } H_{f}(T) & \boldsymbol{x} & \boldsymbol{v} & \boldsymbol{v} \\ & & & \\ \text { Universal shape } g(y) & \boldsymbol{x} & \boldsymbol{x} & \boldsymbol{x} \\ \text { Front location } X_{f}(T) & \boldsymbol{x} & \boldsymbol{x} & \boldsymbol{x} \\ \text { Vertical reach } H_{f}(T) & \boldsymbol{x} & \boldsymbol{x} & \boldsymbol{x}\end{array}$

Universal shape

Front location $X_{f}(T)$

Inlet thickness $H_{i}$

Universal shape

Front location $X_{f}(T)$

Inlet thickness $H_{i}$

Universal shape

Front location $X_{f}(T)$

Inlet thickness $H_{i}$

Universal shape

Front location $X_{f}(T)$

Inlet thickness $H_{i}$

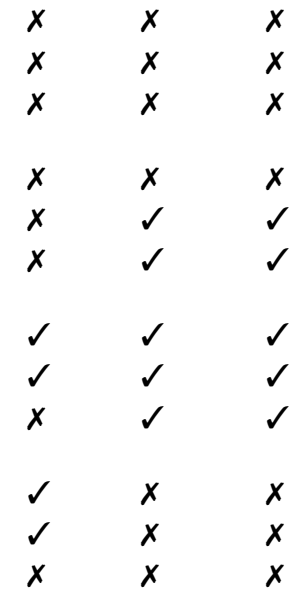

TABLE 3. The influence of dimensionless parameters $N, H_{e}$ and $\Lambda$ on the similarity solutions for the interface shape $H(X, T)$ in the schematic regime diagram (figure 18). Here $N$ is the modified viscosity ratio, $H_{e}$ is the rescaled capillary length and $\Lambda$ is the pore heterogeneity parameter, as defined in table 1 . The "universal shape" in the late-time confined flow limit is defined as the universal functional form of $H / H_{i}$ vs $X / X_{f}$. Here $\checkmark$ indicates that the parameter is relevant, while $\boldsymbol{X}$ indicates that the parameter is irrelevant.

model recovers the sharp-interface case with the viscosity ratio $N$ as the only control parameter, which determines the shock $(\mathrm{S})$ and rarefaction $(\mathrm{R})$ solutions in $§ 3.4 .2$. With capillary effects, the interface shape evolves into either a modified shock (MS) or a compound wave $(\mathrm{CW})$ solution, with the front location $X_{f}$ depending on $N, H_{e}$ and $\Lambda$ and the inlet thickness $H_{i}<1$ depending on $H_{e}$ and $\Lambda$ from (3.27).

Once the interface shape $H(X, T)$ is obtained, the saturation field can be calculated based on (3.10) and is only dependent on $H_{e}$ and $\Lambda$. The influence of $\Lambda$ and $H_{e}$ has already been shown in figure 4 , with $H=1 / 2$ as an example. We note that the calculation demonstrates that the saturation field approaches the sharp-interface limit as $\Lambda \rightarrow \infty$, the limit of a monodispersed medium, or $H_{e} \rightarrow 0^{+}$, where the capillary entry pressure becomes negligible. 
Geophysical data:

Permeability $k$

Porosity $\phi$

Thickness $h_{0}$

$\mathrm{CO}_{2}$ density $\rho_{n}$

Brine density $\rho_{w}$

$\mathrm{CO}_{2}$ viscosity $\mu_{n}$

Brine viscosity $\mu_{w}$

Injection rate $q$

Length of horizontal well $l_{w}$

$\begin{array}{lll}{[\mathrm{mD}]} & 2.0 \times 10^{3} & 20 \\ {[-]} & 0.36 & 0.17 \\ {[\mathrm{~m}]} & 11.3 & 20 \\ {\left[\mathrm{~kg} / \mathrm{m}^{3}\right]} & 760 & 678 \\ {\left[\mathrm{~kg} / \mathrm{m}^{3}\right]} & 1.02 \times 10^{3} & 978 \\ {[\mathrm{mPa} \cdot \mathrm{s}]} & 0.060 & 0.056 \\ {[\mathrm{mPa} \cdot \mathrm{s}]} & 0.80 & 0.32 \\ {[\mathrm{Mt} / \mathrm{yr}]} & 1.0 & 0.30 \\ {[\mathrm{~km}]} & 4.1 & 1.0\end{array}$

Two-phase flow properties:

Irreducible brine saturation $S_{w i}$

End-point relative permeability $k_{r n 0}$

Capillary entry pressure $p_{e}$

Characteristic scales:

Capillary length $h_{c}$

Time scale $t_{c}$

Length scale $x_{c}$

Dimensionless control parameters:

Modified viscosity ratio $N$

Pore size distribution $\Lambda$

Rescaled capillary length $H_{e}$

Sharp interface model:

$\begin{array}{ll}{[\mathrm{m}]} & 8.3 \\ {[\mathrm{yr}]} & 1.4 \\ {[\mathrm{~m}]} & 12\end{array}$

72

0.024

3.5

0.66

2

3.6

$\begin{array}{lll}{[-]} & 1.5 & 0.66 \\ {[-]} & 2 & 2 \\ {[-]} & 0.74 & 3.6\end{array}$

$\begin{array}{llll}\text { Viscosity ratio } M & {[-]} & 13 & 5.7 \\ \text { Time scale } t_{c s} & {[\mathrm{yr}]} & 14 & 0.23 \\ \text { Length scale } x_{c s} & {[\mathrm{~m}]} & 1.1 \times 10^{3} & 30\end{array}$

TABLE 4. $\mathrm{CO}_{2}$ geological sequestration projects at Sleipner and In Salah. The geophysical and two-phase flow data are taken from Bennion \& Bachu (2005), Golding et al. (2011), Guo et al. (2016a), Yu et al. (2017) and Cowton et al. (2018). For the Sleipner project, the length of the horizontal well is taken from EPA (2010), while for the In Salah project, only the injection well KB-501 is considered with length $1 \mathrm{~km}$ (Petropoulos \& Srivastava 2016). $S_{w i}$ is taken as the average value of four sandstone samples in Krevor et al. (2012). The capillary entry pressure is estimated as $p_{e} \approx \gamma / k^{1 / 2}$, where $k$ is the permeability and $\gamma \approx 30 \mathrm{mN} / \mathrm{m}$ is the interfacial tension between supercritical $\mathrm{CO}_{2}$ and brine (Bachu \& Bennion 2009). The time and length scales $\left(t_{c s}\right.$ and $\left.x_{c s}\right)$ in the sharp interface model are defined in $(2.11 b, c)$, respectively, in Zheng et al. $(2015 a)$. 

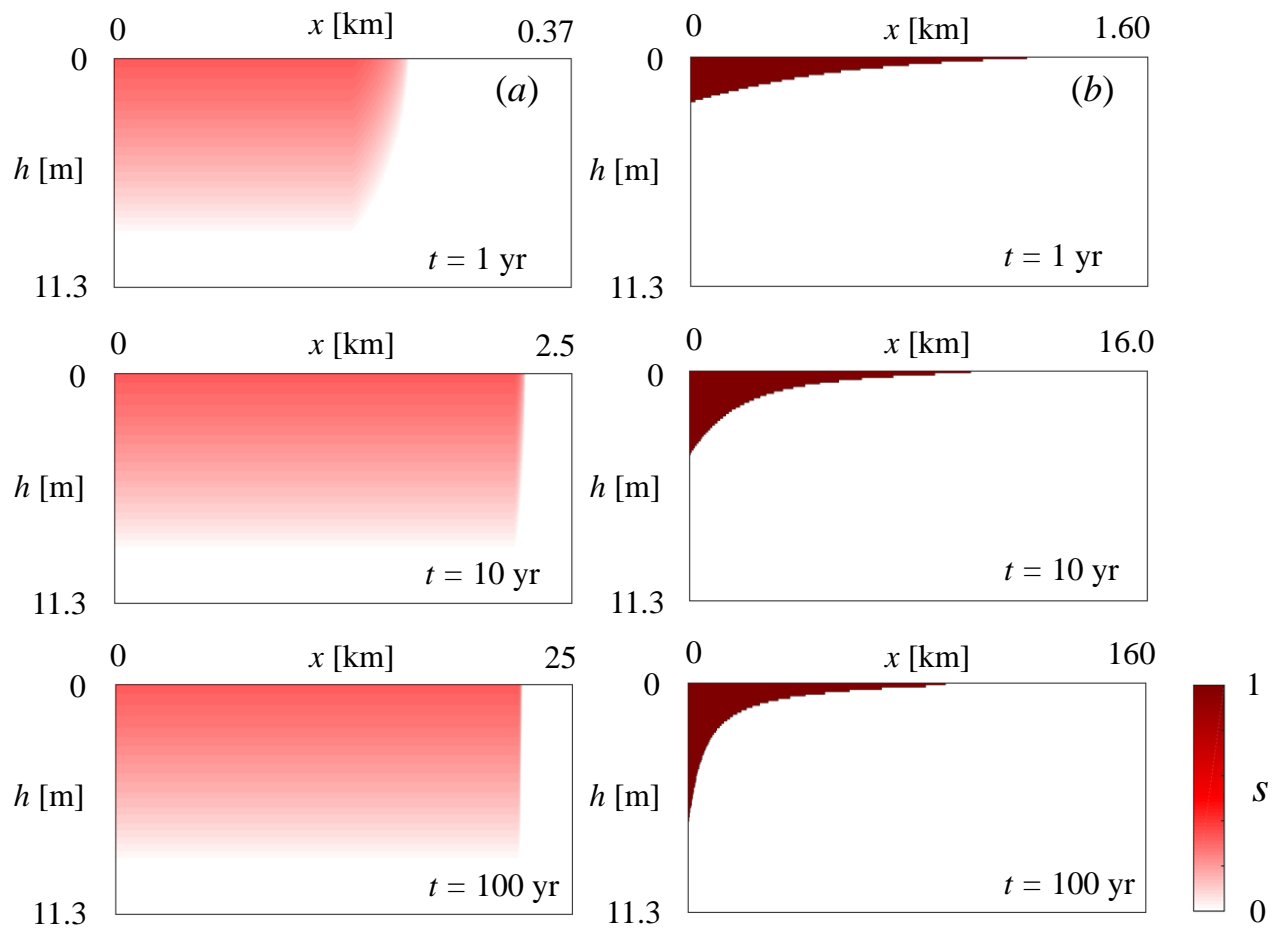

Figure 19. The distribution of supercritical $\mathrm{CO}_{2}$ in the saline aquifer at the Sleipner site at $t=\{1,10,100\}$ yr: $(a)$ shows simulation results based on the current model of partially saturating flows; the $\mathrm{CO}_{2}$ front reaches $x_{f} \approx\{0.239,2.23,22.1\} \mathrm{km}$ and covers a total area of $A \approx\{1.99,19.5,194\} \times 10^{-3} \mathrm{~km}^{2}$ at the corresponding times. (b) shows simulation results based on the sharp interface model (Pegler et al. 2014; Zheng et al. 2015a); the $\mathrm{CO}_{2}$ front arrives at $x_{f} \approx\{1.18,9.85,90.2\} \mathrm{km}$ and covers an area of $A \approx\{0.89,8.9,89\} \times 10^{-3} \mathrm{~km}^{2}$ at identical times.

\section{Implications to $\mathrm{CO}_{2}$ geological sequestration}

While the present study is applicable to many confined, two-phase flows in porous media, we briefly discuss the implication of the current study to the geological sequestration of $\mathrm{CO}_{2}$. We use representative properties of two practical $\mathrm{CO}_{2}$ sequestration projects, the Sleipner project in Norway and the In Salah project in Algeria, as summarized in table 4. We compare the evolution of the injected supercritical $\mathrm{CO}_{2}$ in the saline aquifer computed using two different models for fluid injection into a confined porous layer: The sharp interface model (Pegler et al. 2014; Zheng et al. 2015a) and the model of twophase flows presented here. The main results are summarised in table 5 , including the front loation $x_{f}(t)$, the vertical reach $h_{f}(t)$ and the total area covered by the spreading $\mathrm{CO}_{2}$ current $A(t)$ at different representative times.

We note that the form of the capillary pressure and relative permeability curves can significantly change the model results of partially saturating $\mathrm{CO}_{2}$ flows in a saline aquifer. In the absence of multiphase flow properties for the specific sites, we use the laboratory measurements from Bennion \& Bachu (2005) for $\mathrm{CO}_{2}$ in Ellerslie Sandstone samples in the Alberta Basin, Canada. A review of various models for consolidated rocks and more recent studies can be found in Li \& Horne (2006) and Krevor et al. (2012). The main focus of the calculation in this section is to provide an illustrative example which 

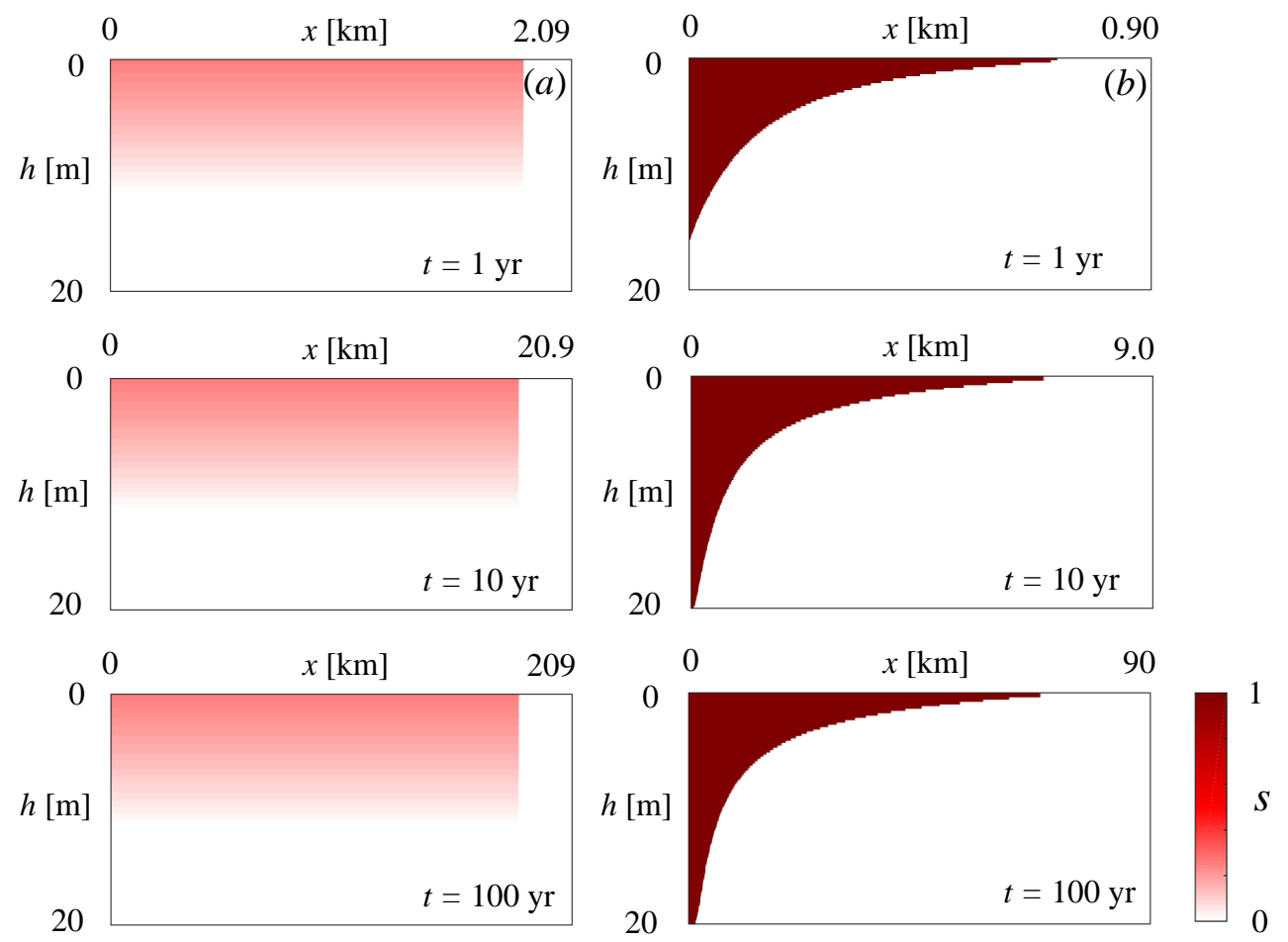

FIgURE 20. The distribution of supercritical $\mathrm{CO}_{2}$ at the In Salah site at $t=\{1,10,100\}$ yr: $(a)$ shows simulation results based on the current model of partially saturating flows; the $\mathrm{CO}_{2}$ front reaches $x_{f} \approx\{1.85,18.5,185\} \mathrm{km}$ and covers an area of $A \approx\{21.4,211,2110\} \times 10^{-3} \mathrm{~km}^{2}$ at the corresponding times. $(b)$ shows simulation results based on the sharp interface model (Pegler et al. 2014; Zheng et al. 2015a); the $\mathrm{CO}_{2}$ front arrives at $x_{f} \approx\{0.716,6.86,68.4\} \mathrm{km}$ and covers a total area of $A \approx\{2.6,26,260\} \times 10^{-3} \mathrm{~km}^{2}$ at identical times.

demonstrates, in principle, how capillary forces and pore-size distribution can modify the dynamic behaviour of the $\mathrm{CO}_{2}$ current such as the evolution of the interface shape, the front location and the total area covered by the injected $\mathrm{CO}_{2}$.

The evolution of the distribution of the injected supercritical $\mathrm{CO}_{2}$ in the saline aquifer is shown at three different times, $t=\{1,10,100\}$ years, for the Sleipner project (figure 19) and In Salah project (figure 20). For both projects, the distribution of $\mathrm{CO}_{2}$ behaves very differently from the prediction of the sharp interface model, considering the effects of the capillary forces and the pore size distribution. Neglecting the effects of capillary forces and fluid mixing, the sharp interface model predicts that the interface shape between the $\mathrm{CO}_{2}$ current and brine approaches a rarefaction solution as time progresses, while the current model of two-phase partially saturating flows indicates that the interface shape approaches the modified shock solution, with an inlet height of $8.8 \mathrm{~m}$ at the Sleipner site and $11.4 \mathrm{~m}$ at the In Salah site. The numerical solutions clearly demonstrate such behaviours.

One key aspect is the location of the propagating front of the injected $\mathrm{CO}_{2}$. The effects of capillary forces and pore size distribution impose different influence for the Sleipner and In Salah projects. The numerical simulation shows that at Sleipner, $\mathrm{CO}_{2}$ spreads slower in the partially saturating flow model than the sharp-interface model. The 


\begin{tabular}{lllll}
\hline Items & Unit & Sleipner (SI) & Sleipner (UF) & In Salah (SI)
\end{tabular}

Front location:

$\begin{array}{llllll}\text { Year } 1 & {[\mathrm{~km}]} & 1.18 & 0.239 & 0.716 & 1.85 \\ \text { Year } 10 & {[\mathrm{~km}]} & 9.85 & 2.23 & 6.86 & 18.5 \\ \text { Year } 100 & {[\mathrm{~km}]} & 90.2 & 22.1 & 68.4 & 185\end{array}$

Vertical reach:

$\begin{array}{llllll}\text { Year } 1 & {[\mathrm{~m}]} & 2.2 & 8.8 & 16 & 11.4 \\ \text { Year } 10 & {[\mathrm{~m}]} & 4.1 & 8.8 & 20 & 11.4 \\ \text { Year } 100 & {[\mathrm{~m}]} & 7.1 & 8.8 & 20 & 11.4\end{array}$

Area of $\mathrm{CO}_{2}$ :

$\begin{array}{llllll}\text { Year } 1 & {\left[\mathrm{~km}^{2}\right]} & 8.9 \times 10^{-4} & 1.99 \times 10^{-3} & 2.6 \times 10^{-3} & 2.14 \times 10^{-2} \\ \text { Year } 10 & {\left[\mathrm{~km}^{2}\right]} & 8.9 \times 10^{-3} & 1.95 \times 10^{-2} & 2.6 \times 10^{-2} & 2.11 \times 10^{-1} \\ \text { Year 100 } & {\left[\mathrm{km}^{2}\right]} & 8.9 \times 10^{-2} & 1.94 \times 10^{-1} & 2.6 \times 10^{-1} & 2.11\end{array}$

TABLE 5. Implications to $\mathrm{CO}_{2}$ geological sequestration projects at the Sleipner and In Salah sites: Predictions for the location of the spreading front $\left(x_{f}(t)\right)$, the vertical reach $h_{f}(t)$ and total area covered by the $\mathrm{CO}_{2}$ current $(A(t))$ from two different models. "SI" represents the sharp interface model (Pegler et al. 2014; Zheng et al. 2015a) and "UF" represents the partially saturating flow model (current study).

front location reaches $x_{f} \approx\{0.239,2.23,22.1\} \mathrm{km}$ at $t=\{1,10,100\}$ years, compared with $x_{f} \approx\{1.18,9.85,90.2\} \mathrm{km}$ based on the sharp interface model. In comparison, at the In Salah site, the partially saturating $\mathrm{CO}_{2}$ front spreads much faster and reaches $x_{f} \approx\{1.85,18.5,185\} \mathrm{km}$ at the $t=\{1,10,100\}$ years, while the sharp interface model predicts $x_{f} \approx\{0.716,6.86,68.4\} \mathrm{km}$ at the corresponding times. We note that at the In Salah site, the capillary length $h_{e}=72 \mathrm{~m}$ is much greater than that at the Sleipner site where $h_{e}=8.3 \mathrm{~m}$ and hence the average saturation of $\mathrm{CO}_{2}$ is smaller in the partially saturating $\mathrm{CO}_{2}$ current and the front spreads faster.

The effect of capillary forces, as exemplified by our partially saturated flow formulation, is an increased efficiency of trapping. The volume of reservoir rock contacted by the current, known as the sweep efficiency, affects the rates of both dissolution and capillary trapping. In our 2D formulation, this may be expressed as a difference on the total area $A$ (in the plane of the simulation) covered by the $\mathrm{CO}_{2}$ current. As exemplified by the profiles in figures 19 and 20, the sweep efficiency of the capillary currents is improved at both Sleipner and In Salah. At the Sleipner site, we obtain $A \approx\{1.99,19.5,194\} \times 10^{-3}$ $\mathrm{km}^{2}$ from the two phase model at $t=\{1,10,100\}$ years, which is an increase from $A \approx\{0.89,6.9,89\} \times 10^{-3} \mathrm{~km}^{2}$ from the sharp interface model. At the In Salah site, the two phase model predicts that $A \approx\{21.4,211,2110\} \times 10^{-3} \mathrm{~km}^{2}$ at $t=\{1,10,100\}$ years, which is also a significant increase from $A \approx\{2.6,26,260\} \times 10^{-3} \mathrm{~km}^{2}$ from the sharp interface model. Therefore, at both sites, the effects of capillary forces suggest an increase in the area covered by the $\mathrm{CO}_{2}$ current, and hence an increase of the amount of $\mathrm{CO}_{2}$ that can be trapped from dissolution into brine. 


\section{Summary and conclusions}

We have investigated the behaviour of two-phase partially saturating flows resulting from fluid injection into a confined porous layer, and focus on the evolution of the fluidfluid interface, the location of the propagating fronts and the saturation field of the injected and displaced fluids. We derive an evolution equation to describe the dynamics of the interface, from which the saturation field can be subsequently calculated. We also provide an example calculation to demonstrate the transition from early-time unconfined to late-time confined flows, and we obtain six flow regimes in which the current exhibits different self-similar spreading behaviours (figure 18). Three of these regimes (C, MS and $\mathrm{CW}$ in figure 18) are due to the action of capillary forces in the polydispersed porous medium and are different from those in the sharp-interface model (B, S and R in figure 18) (Pegler et al. 2014; Zheng et al. 2015a). It is of practical interests to explore the implications to the geological $\mathrm{CO}_{2}$ sequestration, which we briefly discussed in $\S 6$ before we close the paper. Our example calculations suggest that the capillary forces can significantly modify the evolution of the front location of the $\mathrm{CO}_{2}$ current and the efficiency of sweeping and trapping.

\section{Acknowledgement}

This work is funded by the Natural Environment Research Council (NERC) through research grant NE/N016084/1. JAN also acknowledge support from a Royal Society University Research Fellowship. We also thank H. E. Huppert for helpful discussions and two anonymous referees for their comments on the paper.

\section{Appendix A. Evaluating the integrals $I_{n}(h)$ and $I_{w}(h)$}

We evaluate the integrals $I_{n}(h)$ and $I_{w}(h)$, given that the relative permeability functions $k_{n}(s)$ and $k_{w}(s)$ are in power-law forms, i.e., equation $(2.7 a, b)$. First, the vertical integration of the wetting-phase relative permeability function $k_{w}(s)$ provides

$$
I_{w}(h)= \begin{cases}h_{0}-h+\frac{h_{e}}{1-\beta \Lambda}\left[1-\left(1+\frac{h}{h_{e}}\right)^{1-\beta \Lambda}\right], & \beta \Lambda \neq 1 \\ h_{0}-h-h_{e} \log \left(1+\frac{h}{h_{e}}\right), & \beta \Lambda=1 .\end{cases}
$$

The vertical integration of the non-wetting-phase relative permeability function $k_{n}(s)$ can also be obtained explicitly for special values of $\alpha$ in equation $(2.7 a)$. For example, when $\alpha=1$, we have

$$
I_{n}(h)= \begin{cases}k_{r n 0}\left(h+\frac{h_{e}}{1-\Lambda}\left[1-\left(1+\frac{h}{h_{e}}\right)^{1-\Lambda}\right]\right), & \Lambda \neq 1 \\ k_{r n 0}\left[h+h_{e} \log \left(1+\frac{h}{h_{e}}\right)\right], & \Lambda=1 .\end{cases}
$$



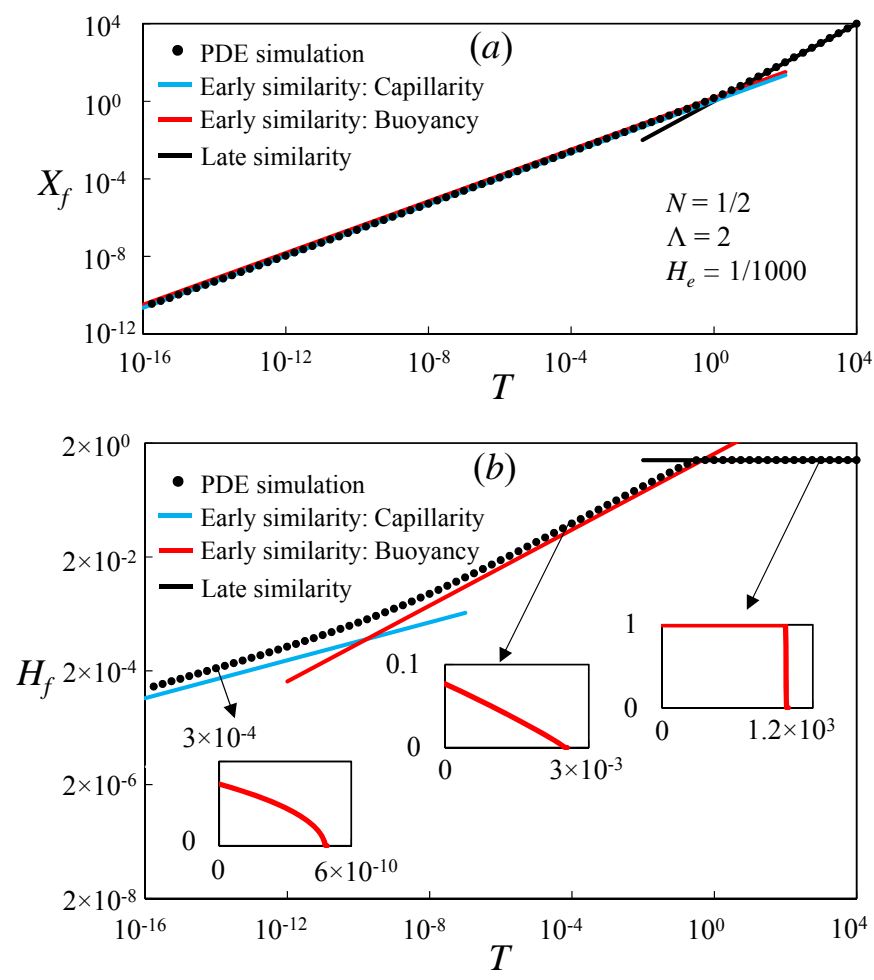

FIGURE 21. Evolution for the front location $X_{f}(T)$ in $(a)$ and vertical reach $H_{f}(T)$ in $(b)$ for $N=1 / 2, \Lambda=2$ and $H_{e}=1 / 1000$. PDE numerical solutions are shown as dots, while the early-time and late-time self-similar solutions are shown as straight lines. The insets in $(b)$ are the profiles at different representative times $T=\left\{10^{-14}, 10^{-4}, 10^{3}\right\}$ from PDE numerical solutions.

When $\alpha=2$, which excellently fits the experimental data from a $\mathrm{CO}_{2}$-Ellerslie standstone system (Bennion \& Bachu 2005), we obtain

$$
I_{n}(h)= \begin{cases}k_{r n 0}\left(h+\frac{2 h_{e}}{1-\Lambda}\left[1-\left(1+\frac{h}{h_{e}}\right)^{1-\Lambda}\right]-\frac{h_{e}}{1-2 \Lambda}\left[1-\left(1+\frac{h}{h_{e}}\right)^{1-2 \Lambda}\right]\right), & \Lambda \neq 1,1 / 2 ; \\ k_{r n 0}\left(h-2 h_{e} \log \left(1+\frac{h}{h_{e}}\right)+h_{e}\left[1-\left(1+\frac{h}{h_{e}}\right)^{-1}\right]\right), & \Lambda=1 ; \\ k_{r n 0}\left(h+h_{e} \log \left(1+\frac{h}{h_{e}}\right)+4 h_{e}\left[1-\left(1+\frac{h}{h_{e}}\right)^{1 / 2}\right]\right), & \Lambda=1 / 2 .\end{cases}
$$

The resulting expressions (A 1) and (A 3) are then substituted into the evolution equation (2.16) to obtain a revised form for further analyses in $\S 3$.

\section{Appendix B. Transition dynamics: $N=1 / 2$}

In the sharp interface limit, viscosity ratios $N<1$ result in a shock solution in the late time period. We set $N=1 / 2, \Lambda=2$ and $H_{e}=\{1 / 1000,1 / 5\}$ in the numerical solutions to demonstrate the capillary effects on the evolution of the front location and interface shape, as shown in figures 21-23.

When $H_{e}=1 / 1000$, the numerical solution starts from a capillarity similarity solution 

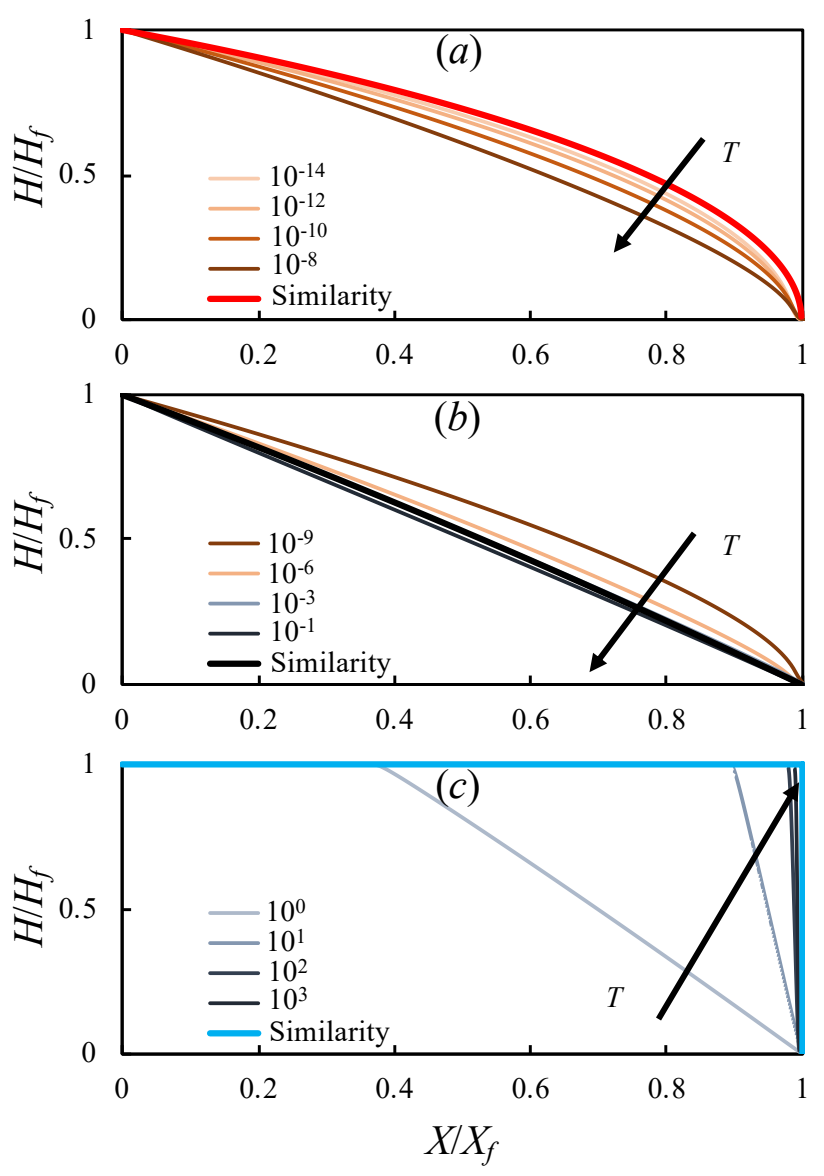

Figure 22. Evolution for the rescaled shapes with $N=1 / 2, \Lambda=2$ and $H_{e}=1 / 1000$. The numerical simulation departs from the capillarity similarity solution of (3.15) in the early-time period in $(a)$, approaches the buoyancy similarity solution (3.22) at intermediate times in $(b)$, before eventually approaches the confined similarity solution in the late-time period in $(c)$.

at early times (figures 21, 22a). Then, the numerical solution departs from the capillarity similarity solution while approaches the buoyancy similarity solution at intermediate times (figures 21, 22b). At late times, the numerical solution approaches a shock solution (figures 21, 22c). In comparison, when $H_{e}=1 / 5$, the numerical solution does not show the buoyancy similarity solution at intermediate times, while it approaches a modified shock solution at late times (figure $23 a, b, d$ ).

\section{REFERENCES}

Acton, J. M., Huppert, H. E. \& Worster, M. G. 2001 Two-dimensional viscous gravity currents flowing over a deep porous medium. J. Fluid Mech. 440, 359-380.

Bachu, S. \& Bennion, D. B. 2009 Interfacial tension between $\mathrm{CO}_{2}$, freshwater, and brine in the range of pressure from (2 to 27) $\mathrm{MPa}$, temperature from $(20$ to 125$){ }^{\circ} \mathrm{C}$, and water salinity from (0 to 334000$) \mathrm{mg} \cdot \mathrm{L}^{-1}$. J. Chem. Eng. Data 54, 765-775.

Barenblatt, G. I. 1952 On some unsteady fluid and gas motions in a porous medium (in Russian). Prikl. Mat. Mekh. (PMM) 16, 67-78.

Bear, J. 1972 Dynamics of Fluids in Porous Media. Elsevier.

Bennion, B. \& BACHU, S. 2005 Relative permeability characteristics for supercritical $\mathrm{CO}_{2}$ 

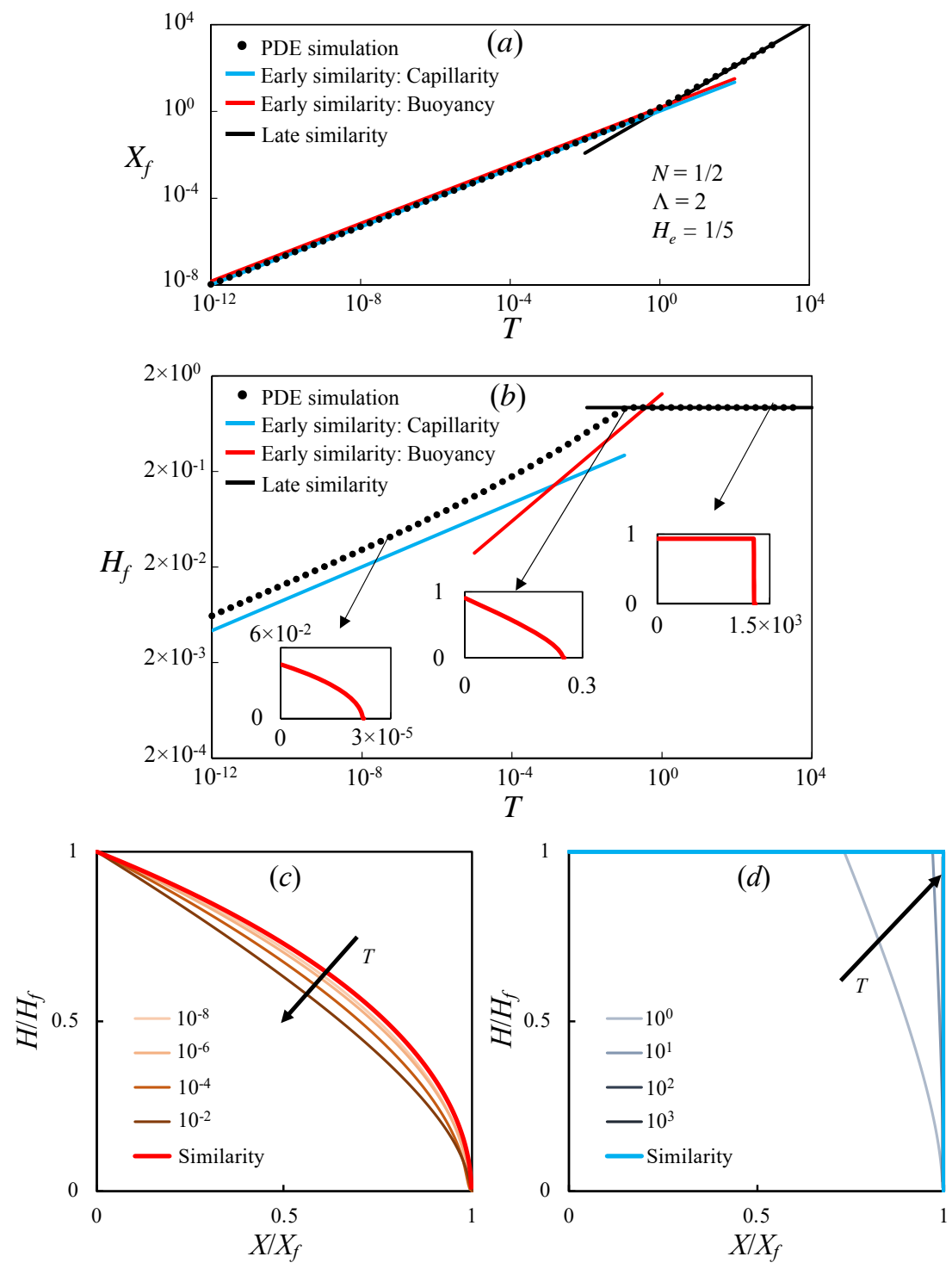

Figure 23. Evolution for the front location $X_{f}(T)$ in $(a)$, vertical reach $H_{f}(T)$ in $(b)$ and profile shapes in $(c, d)$ for $N=1 / 2, \Lambda=2$ and $H_{e}=1 / 5$. In $(a, b)$, the numerical solutions are shown as dots, while the early-time and late-time self-similar solutions are shown as straight lines. The insets in $(b)$ are the profiles at different representative times $T=\left\{10^{-7}, 10^{-1}, 10^{3}\right\}$ from numerical solutions. In $(c, d$, the numerical solutions depart from the capillarity similarity solution of (3.15) in the early-time period in $(c)$, while they approach the confined similarity solution (modified shock) in the late-time period in $(d)$ )

displacing water in a variety of potential sequestration zones in western Canada sedimentary basin. In SPE Annual Technical Conference and Exhibition. Dallas, Texas (SPE 99325).

BoussinesQ, J. V. 1904 Recherches theoretique sur l'ecoulement des nappes d'eau infiltrees dans le sol et sur le debit des sources. J. Math. Pure Appl. 10, 5-78.

Brooks, R. H. \& Corey, A. T. 1964 Hydraulic properties of porous media. In Hydrology Papers 3. Colorado State University. 
Buckley, S. E. \& Leverett, M. C. 1942 Mechanism of fluid displacement in sands. Trans. AIME 146, 107-116.

Cowton, L. R., Neufeld, J. A., White, N. J., Bickle, M. J., Williams, G. A., White, J. C. \& Chadwick, R. A. 2018 Benchmarking of vertically-integrated $\mathrm{CO}_{2}$ flow simulations at the Sleipner Field, North Sea. Earth Planet. Sci. Lett 491, 121-133.

de Gennes, P.-G., Brochard-Wyart, F. \& Quere, D. 2004 Capillarity and Wetting Phenomena. Springer-Verlag New York.

EPA 2010 Issues concerning the use of horizontal wells in the injection of carbon dioxide for geologic sequestration. Tech. Rep.. Office of Water, United States Environmental Protection Agency.

FArcas, A. \& Woods, A. W. 2009 The effect of drainage on the capillary retention of $\mathrm{CO}_{2}$ in a layered permeable rock. J. Fluid Mech. 618, 349-359.

Gasda, S. E., Bachu, S. \& Celia, M. A. 2004 Spatial characterization of the location of potentially leaky wells penetrating a deep saline aquifer in a mature sedimentary basin. Environ. Geol. 46, 707-720.

Gasda, S. E., Nordbotten, J. M. \& Celia, M. A. 2009 Vertical equilibrium with sub-scale analytical methods for geological $\mathrm{CO}_{2}$ sequestration. Comput. Geosci. 13, 469-481.

Golding, M. J., Huppert, H. E. \& Neufeld, J. A. 2013 The effects of capillary forces on the axisymmetric propagation of two-phase, constant-flux gravity currents in porous media. Phys. Fluids 25, 036602.

Golding, M. J., Huppert, H. E. \& Neufeld, J. A. 2017 Two-phase gravity currents resulting from the release of a fixed volume of fluid in a porous medium. J. Fluid Mech. 832, 550-577.

Golding, M. J., Neufeld, J. A., Hesse, M. A. \& Huppert, H. E. 2011 Two-phase gravity currents in porous media. J. Fluid Mech. 678, 248-270.

Guo, B., Zheng, Z., Bandilla, K. W., Celia, M. A. \& Stone, H. A. $2016 a$ Flow regime analysis for geologic $\mathrm{CO}_{2}$ sequestration and other subsurface fluid injections. Int. J. Greenh. Gas Con. 53, 284-291.

Guo, B., Zheng, Z., Celia, M. A. \& Stone, H. A. $2016 b$ Axisymmetric flows from fluid injection into a confined porous medium. Phys. Fluids 28, 022107.

Hesse, M. A., OrR JR, F. M. \& TChelepi, H. A. 2008 Gravity currents with residual trapping. J. Fluid Mech. 611, 35-60.

Hesse, M. A. \& Woods, A. W. 2010 Buoyant disposal of $\mathrm{CO}_{2}$ during geological storage. Geophys. Res. Lett. 37, L01403.

Huppert, H. E. \& Neufeld, J. A. 2014 The fluid mechanics of carbon dioxide sequestration. Annu. Rev. Fluid Mech. 46, 255-272.

Huppert, H. E. \& Woods, A. W. 1995 Gravity driven flows in porous layers. J. Fluid Mech. 292, 55-69.

Juanes, R., MacMinn, C. W. \& Szulczewski, M. L. 2010 The footprint of the $\mathrm{CO}_{2}$ plume during carbon dioxide storage in saline aquifers: storage efficiency for capillary trapping at the basin scale. Trans. Porous Med. 82, 19-30.

Kochina, I. N., Mikhailov, N. N. \& Filinov, M. V. 1983 Groundwater mound damping. Int. J. Eng. Sci. 21, 413-421.

Krevor, S. C. M., Pini, R., Zuo, L. \& Benson, S. M. 2012 Relative permeability and trapping of $\mathrm{CO}_{2}$ and water in sandstone rocks at reservoir conditions. Water Resour. Rea. 48, W02532.

Kurganov, A. \& TADMOR, E. 2000 New high-resolution central schemes for nonlinear conservation laws and convection-diffusion equations. J. Comput. Phys. 160, 241-282.

LAKe, L. W. 1989 Enhanced Oil Recovery. Prentice Hall, Englewood Cliffs, NJ.

LeVeque, R. J. 2002 Finite Volume Methods for Hyperbolic Problems. Cambridge University Press.

Leverett, M. C. 1941 Capillary behavior in porous solids. Trans. AIME 142, 152-169.

LI, K. \& Horne, R. N. 2006 Comparison of methods to calculate relative permeability from capillary pressure in consolidated water-wet porous media. Water Resour. Res 42, W06405.

Liu, Y., Zheng, Z. \& Stone, H. A. 2017 The influence of capillary effects on the drainage of a viscous gravity current into a deep porous medium. J. Fluid Mech. 817, 514-559.

Lyle, S., Huppert, H. E., Hallworth, M., Bickle, M. \& Chadwick, A. 2005 Axisymmetric gravity currents in a porous medium. J. Fluid Mech. 543, 293-302. 
MacMinn, C. W., Szulczewski, M. L. \& Juanes, R. $2010 \mathrm{CO}_{2}$ migration in saline aquifers. Part 1. Capillary trapping under slope and groundwater flow. J. Fluid Mech. 662, 329-351.

MacMinn, C. W., Szulczewski, M. L. \& Juanes, R. $2011 \mathrm{CO}_{2}$ migration in saline aquifers. Part 2. Combined capillary and solubility trapping. J. Fluid Mech. 688, 321-351.

Neufeld, J. A., Vella, D. \& Huppert, H. E. 2009 The effect of a fissure on storage in a porous medium. J. Fluid Mech. 639, 239-259.

Neufeld, J. A., Vella, D., Huppert, H. E. \& Lister, J. R. 2011 Leakage from gravity currents in a porous medium. Part 1. a localized sink. J. Fluid Mech. 666, 391-413.

Nilsen, H. M., Lie, K.-A. \& Andersen, O. 2016 Full-implicit simulation of verticalequilibrium models with hysteresis and capillary fringe. Comput. Geosci. 20, 49-67.

Nordbotten, J. M. \& Celia, M. A. 2006 Similarity solutions for fluid injection into confined aquifers. J. Fluid Mech. 561, 307-327.

Nordbotten, J. M. \& DAhle, H. K. 2011 Impact of the capillary fringe in vertically integrated models for $\mathrm{CO}_{2}$ storage. Water Resour. Rea. 47, W02537.

Pegler, S. S., Huppert, H. E. \& Neufeld, J. A. 2014 Fluid injection into a confined porous layer. J. Fluid Mech. 745, 592-620.

Petropoulos, G. \& Srivastava, P. 2016 Sensitivity analysis in earth observation modelling. Elsevier.

Phillips, O. M. 1991 Flow and Reactions in Permeable Rocks. Cambridge University Press.

Pritchard, D., Woods, A. W. \& HogG, A. J. 2001 On the slow draining of a gravity current moving through a layered permeable medium. J. Fluid Mech. 444, 23-47.

Taghavi, S. M., Martinez, D. M. \& Frigaard, I. A. 2009 Buoyancy-dominated displacement flows in near-horizontal channels: the viscous limit. J. Fluid Mech. 639, 1-35.

Vella, D., Neufeld, J. A., Huppert, H. E. \& Lister, J. R. 2011 Leakage from gravity currents in a porous medium. Part 2. a line sink. J. Fluid Mech. 666, 414-427.

Woods, A. W. \& FARCAS, A. 2009 Capillary entry pressure and the leakage of gravity currents through a sloping layered permeable rock. J. Fluid Mech. 618, 361-379.

Yu, Y. E., Zheng, Z. \& Stone, H. A. 2017 Flow of a gravity current in a porous medium accounting for drainage from a permeable substrate and an edge. Phys. Rev. Fluids 2, 074101.

Zheng, Z., Guo, B., Christov, I. C., Celia, M. A. \& Stone, H. A. $2015 a$ Flow regimes for fluid injection into a confined porous medium. J. Fluid Mech. 767, 881-909.

Zheng, Z., Ronge, L. \& Stone, H. A. $2015 b$ Viscous fluid injection into a confined channel. Phys. Fluids 27, 062105.

Zheng, Z., Shin, S. \& Stone, H. A. $2015 c$ Converging gravity currents over a porous substrate. J. Fluid Mech. 778, 669-690.

Zheng, Z., Soh, B., Huppert, H. E. \& Stone, H. A. 2013 Fluid drainage from the edge of a porous reservoir. J. Fluid Mech. 718, 558-568. 